\title{
The Evolution of States in a Spatial Population Model
}

\author{
Yuri Kondratiev ${ }^{1,2}$. Yuri Kozitsky ${ }^{3}$
}

Received: 8 February 2016 / Published online: 7 March 2016

(C) The Author(s) 2016. This article is published with open access at Springerlink.com

\begin{abstract}
The evolution of states in a spatial population model is studied. The model describes an infinite system of point entities in $\mathbb{R}^{d}$ which reproduce themselves at distant points (disperse) and die with rate that includes a competition term. The system's states are probability measures on the space of configurations, and their evolution is obtained from a hierarchical chain of differential equations for the corresponding correlation functions derived from the Fokker-Planck equation for the states. Under natural conditions imposed on the model parameters it is proved that the correlation functions evolve in a scale of Banach spaces in such a way that at each moment of time the correlation function corresponds to a unique sub-Poissonian state. Some further properties of the evolution of states constructed in this way are described.
\end{abstract}

Keywords Markov evolution - Configuration space - Stochastic semigroup - Sun-dual semigroup · Individual-based model · Correlation function · Scale of Banach spaces

Mathematics Subject Classification $60 \mathrm{~J} 80 \cdot 92 \mathrm{D} 25 \cdot 82 \mathrm{C} 22$

\section{Introduction}

\subsection{Posing}

The development of a mathematical theory of complex living systems is a challenging task of modern mathematics [4]. In many cases of such systems, one deals with birth-and-death

Yuri Kozitsky

jkozi@hektor.umcs.lublin.pl

Yuri Kondratiev

kondrat@math.uni-bielefeld.de

1 Fakutät für Mathematik, Universität Bielefeld, 33615 Bielefeld, Germany

2 Interdisciplinary Center for Complex Systems, Dragomanov University, Kiev, Ukraine

3 Instytut Matematyki, Uniwersytet Marii Curie-Skłodowskiej, 20-031 Lublin, Poland 
processes the theory of which traces back to works by Kolmogorov and Feller, see [9, Chapter XVII] and e.g. $[16,30]$ for a more recent account on the related concepts and results. In the simplest models of this kind, the system is finite and the state space is $\mathbb{N}_{0}:=\mathbb{N} \cup\{0\}$. Then the time evolution of the probability of having $n$ particles in the system is obtained from the Kolmogorov equation in which the generator is a tridiagonal infinite matrix containing the birth and death rates $\lambda_{n}$ and $\mu_{n}$, respectively. If their increase is controlled by affine functions of $n$, the evolution is obtained with the help of a stochastic semigroup, see e.g. [3,22] and the papers quoted in these works. However, if $\lambda_{n}$ and $\mu_{n}$ increase faster than $n$, one would not expect that the evolution takes place, for all $t>0$, in one and the same Banach space and thus is described by a $C_{0}$-semigroup of operators acting in this space. For infinite systems, the situation is much more complex as the very definition of the Kolmogorov equation cannot be performed directly (for $\lambda_{\infty}$ and $\mu_{\infty}$ are infinite in such cases). The main result of the present paper consists in constructing the evolution of states of an infinite birth-and-death system of particles placed in $\mathbb{R}^{d}$ with 'rates' that roughly speaking increase as $n^{2}$, cf. (3.9) below. This evolution takes place in an ascending sequence of Banach spaces and is obtained by a method developed in the paper. To the best of our knowledge, this is the first construction of this type.

We continue, cf. [10,12,13,23], studying the model introduced in [6,7,24]. It describes an infinite evolving population of identical point entities (particles) distributed over $\mathbb{R}^{d}$, $d \geq 1$, which reproduce themselves and die, also due to competition. This is one of the most important individual-based models in studying large ecological communities (e.g. of perennial plants), see [27] and [25, page 1311]. As is now commonly adopted [6,7,27], the appropriate mathematical context for studying models of this kind is provided by the theory of random point fields in $\mathbb{R}^{d}$ in which populations are modeled as point configurations constituting the set

$$
\Gamma=\left\{\gamma \subset \mathbb{R}^{d}:|\gamma \cap \Lambda|<\infty \text { for any compact } \Lambda \subset \mathbb{R}^{d}\right\},
$$

where $|\cdot|$ denotes cardinality. It is equipped with a $\sigma$-field of measurable subsets that allows one to consider probability measures on $\Gamma$ as states of the system. To characterize such states one employs observables - appropriate functions $F: \Gamma \rightarrow \mathbb{R}$. Their evolution is obtained from the Kolmogorov equation

$$
\frac{d}{d t} F_{t}=L F_{t},\left.\quad F_{t}\right|_{t=0}=F_{0}, \quad t>0
$$

where the generator $L$ specifies the model. The states' evolution is then obtained from the Fokker-Planck equation

$$
\frac{d}{d t} \mu_{t}=L^{*} \mu_{t},\left.\quad \mu_{t}\right|_{t=0}=\mu_{0},
$$

related to that in (1.2) by the duality

$$
\int_{\Gamma} F_{0} d \mu_{t}=\int_{\Gamma} F_{t} d \mu_{0}
$$

The generator for the model studied in this paper is

$$
\begin{aligned}
(L F)(\gamma)= & \sum_{x \in \gamma}\left[m+E^{-}(x, \gamma \backslash x)\right][F(\gamma \backslash x)-F(\gamma)] \\
& +\int_{\mathbb{R}^{d}} E^{+}(y, \gamma)[F(\gamma \cup y)-F(\gamma)] d y,
\end{aligned}
$$


where

$$
E^{ \pm}(x, \gamma):=\sum_{y \in \gamma} a^{ \pm}(x-y)
$$

The first summand in (1.4) corresponds to the death of the particle located at $x$ occurring independently at rate $m \geq 0$ (intrinsic mortality) and under the influence of the other particles in $\gamma$ - at rate $E^{-}(x, \gamma \backslash x) \geq 0$ (competition). The second term in (1.4) describes the birth of a particle at $y \in \mathbb{R}^{d}$ occurring at rate $E^{+}(y, \gamma) \geq 0$. In the sequel, we call $a^{-}$and $a^{+}$ competition and dispersal kernels, respectively. The particular case of (1.4) with $a^{-} \equiv 0$ is the continuum contact model studied in [18,21]. Having in mind the results of these works, along with purely mathematical tasks we aim at understanding the ecological consequences of the competition taken into account in (1.4).

The problem of constructing spatial birth and death processes in infinite volume was first studied by Holley and Stroock in their pioneering work [15], where a special case of nearest neighbor interactions on the real line was considered. For more general versions of continuum birth-and-death systems, the few results known by this time were obtained under severe restrictions imposed on the birth and death rates. This relates to the construction of a Markov process in [14], as well as to the result obtained in [12] in the statistical approach (see below). In the present work, we make an essential step forward in studying the model specified in (1.4) assuming only that the kernels $a^{ \pm}$satisfy some rather natural condition.

The set of finite configurations $\Gamma_{0}$ is a measurable subset of $\Gamma$. If $\mu$ is such that $\mu\left(\Gamma_{0}\right)=1$, then the considered system is finite in this state. If $\mu_{0}$ in (1.3) has such a property, the evolution $\mu_{0} \mapsto \mu_{t}$ can be obtained directly from (1.3), see [23]. In this case $\mu_{t}\left(\Gamma_{0}\right)=1$ for all $t>0$. States of infinite systems are mostly such that $\mu\left(\Gamma_{0}\right)=0$, which makes direct solving (1.3) with an arbitrary initial state $\mu_{0}$ rather unaccessible for the method existing at this time, cf. [20]. In this work we continue following the statistical approach $[5,10,12,13,20]$ in which the evolution of states is described by means of the corresponding correlation functions. To briefly explain its essence let us consider the set of all compactly supported continuous functions $\theta: \mathbb{R}^{d} \rightarrow(-1,0]$. For a probability measure $\mu$ on $\Gamma$ its Bogoliubov functional $[11,19]$ is defined as

$$
B_{\mu}(\theta)=\int_{\Gamma} \prod_{x \in \gamma}(1+\theta(x)) \mu(d \gamma),
$$

with $\theta$ running through the mentioned set of functions. For $\pi_{\varkappa}-$ the homogeneous Poisson measure with intensity $\varkappa>0$, (1.6) takes the form

$$
B_{\pi_{\varkappa}}(\theta)=\exp \left(\varkappa \int_{\mathbb{R}^{d}} \theta(x) d x\right) .
$$

In state $\pi_{\varkappa}$, the particles are independently distributed over $\mathbb{R}^{d}$ with density $\varkappa$. The set of sub-Poissonian states $\mathcal{P}_{\mathrm{sP}}$ is then defined as that containing all the states $\mu$ for which $B_{\mu}$ can be continued, as a function of $\theta$, to an exponential type entire function on $L^{1}\left(\mathbb{R}^{d}\right)$. This exactly means that $B_{\mu}$ can be written down in the form

$$
B_{\mu}(\theta)=\sum_{n=0}^{\infty} \frac{1}{n !} \int_{\left(\mathbb{R}^{d}\right)^{n}} k_{\mu}^{(n)}\left(x_{1}, \ldots, x_{n}\right) \theta\left(x_{1}\right) \cdots \theta\left(x_{n}\right) d x_{1} \cdots d x_{n},
$$

where $k_{\mu}^{(n)}$ is the $n$-th order correlation function corresponding to $\mu$. It is a symmetric element of $L^{\infty}\left(\left(\mathbb{R}^{d}\right)^{n}\right)$ for which

$$
\left\|k_{\mu}^{(n)}\right\|_{L^{\infty}\left(\left(\mathbb{R}^{d}\right)^{n}\right)} \leq C \exp (\alpha n), \quad n \in \mathbb{N}_{0},
$$


with some $C>0$ and $\alpha \in \mathbb{R}$. This guarantees that $B_{\mu}$ is of exponential type. One can also consider a wider class of states, $\mathcal{P}_{\text {anal }}$, by imposing the condition that $B_{\mu}$ can be continued to a function on $L^{1}\left(\mathbb{R}^{d}\right)$ analytic in some neighborhood of the origin, see [19]. In that case, the estimate corresponding to (1.8) will contain $n ! C e^{\alpha n}$ in its right-hand side. States $\mu \in \mathcal{P}_{\text {anal }}$ are characterized by strong correlations corresponding to 'clustering'. In the contact model the clustering does take place, see [18,21] and especially [10, Eq. (3.5), page 303]. Namely, in this model for each $t>0$ and $n \in \mathbb{N}$ the correlation functions satisfy the following estimates

$$
\text { const } \cdot n ! c_{t}^{n} \leq k_{t}^{(n)}\left(x_{1}, \ldots, x_{n}\right) \leq \mathrm{const} \cdot n ! C_{t}^{n},
$$

where the left-hand inequality holds if all $x_{i}$ belong to a ball of sufficiently small radius. If the mortality rate $m$ is big enough, then $C_{t} \rightarrow 0$ as $t \rightarrow+\infty$. That is, in the continuum contact model the clustering persists even if the population asymptotically dies out. With this regard, a paramount question about the model (1.4) is whether the competition contained in $L$ can suppress clustering. In short, the answer given in this work is in affirmative provided the competition and dispersal kernels satisfy a certain natural condition. They do satisfy if $a^{-}$is strictly positive in some vicinity of the origin, and $a^{+}$has finite range.

\subsection{Presenting the Result}

(i) Under the condition on the kernels $a^{ \pm}$formulated in Assumption 1 we prove in Theorem 3.3 that the correlation functions evolve $k_{\mu_{0}}^{(n)} \mapsto k_{t}^{(n)}$ in such a way that each $k_{t}^{(n)}$ is the correlation function of a unique sub-Poissonian measure $\mu_{t}$.

(ii) We give examples of the kernels $a^{ \pm}$which satisfy Assumption 1. These examples include kernels of finite range - both short and long dispersals (Proposition 3.7), and also Gaussian kernels (Propositions 3.8).

(iii) For the whole range of values of the intrinsic mortality rate $m$, in Theorem 3.4 we obtain the following bounds for the correlation functions holding for all $t \geq 0$ :

(i) $0 \leq k_{t}^{(n)}\left(x_{1}, \ldots, x_{n}\right) \leq C_{\delta}^{n} \exp \left(n\left(\left\langle a^{+}\right\rangle-\delta\right) t\right), \quad 0 \leq m \leq\left\langle a^{+}\right\rangle$,

(ii) $0 \leq k_{t}^{(n)}\left(x_{1}, \ldots, x_{n}\right) \leq C_{\varepsilon}^{n} e^{-\varepsilon t}, \quad m>\left\langle a^{+}\right\rangle$,

where $\left\langle a^{+}\right\rangle$is the $L^{1}$-norm of $a^{+}, C_{\delta}$ and $C_{\varepsilon}$ are appropriate positive constants, whereas $\delta<m$ and $\varepsilon \in\left(\left\langle a^{+}\right\rangle, m\right)$ take any value in the mentioned sets. By (1.7) these estimates give upper bounds for the type of $B_{\mu_{t}}$. We describe also the pure death case where $\left\langle a^{+}\right\rangle=0$.

More detailed comments and comparison with the previous results on this model are given in Sect. 3.3 below.

\section{The Basic Notions}

A detailed description of various aspects of the mathematical framework of this paper can be found in $[1,5,10,12,13,17,18,21,26]$. Here we present only some of its aspects and indicate in which of the mentioned papers further details can be found. By $\mathcal{B}\left(\mathbb{R}^{d}\right)$ and $\mathcal{B}_{\mathrm{b}}\left(\mathbb{R}^{d}\right)$ we denote the set of all Borel and all bounded Borel subsets of $\mathbb{R}^{d}$, respectively. 


\subsection{The Configuration Spaces}

The space $\Gamma$ defined in (1.1) is endowed with the weakest topology that makes continuous all the maps

$$
\Gamma \ni \gamma \mapsto \sum_{x \in \gamma} f(x), \quad f \in C_{0}\left(\mathbb{R}^{d}\right) .
$$

Here $C_{0}\left(\mathbb{R}^{d}\right)$ stands for the set of all continuous compactly supported functions $f: \mathbb{R}^{d} \rightarrow$ $\mathbb{R}$. The mentioned topology on $\Gamma$ admits a metrization which turns it into a complete and separable metric (Polish) space. By $\mathcal{B}(\Gamma)$ we denote the corresponding Borel $\sigma$-field. For $n \in \mathbb{N}_{0}:=\mathbb{N} \cup\{0\}$, the set of $n$-particle configurations in $\mathbb{R}^{d}$ is

$$
\Gamma^{(0)}=\{\emptyset\}, \quad \Gamma^{(n)}=\{\eta \subset X:|\eta|=n\}, \quad n \in \mathbb{N} .
$$

For $n \geq 1, \Gamma^{(n)}$ can be identified with the symmetrization of the set

$$
\left\{\left(x_{1}, \ldots, x_{n}\right) \in\left(\mathbb{R}^{d}\right)^{n}: x_{i} \neq x_{j} \text {, for } i \neq j\right\},
$$

which allows one to introduce the topology on $\Gamma^{(n)}$ related to the Euclidean topology of $\mathbb{R}^{d}$ and hence the corresponding Borel $\sigma$-field $\mathcal{B}\left(\Gamma^{(n)}\right)$. The set of finite configurations

$$
\Gamma_{0}:=\bigsqcup_{n \in \mathbb{N}_{0}} \Gamma^{(n)}
$$

is endowed with the topology of the disjoint union and with the corresponding Borel $\sigma$-field $\mathcal{B}\left(\Gamma_{0}\right)$. It is a measurable subset of $\Gamma$. However, the topology just mentioned and that induced on $\Gamma_{0}$ from $\Gamma$ do not coincide.

For $\Lambda \in \mathcal{B}_{\mathrm{b}}\left(\mathbb{R}^{d}\right)$, the set $\Gamma_{\Lambda}:=\{\gamma \in \Gamma: \gamma \subset \Lambda\}$ is a Borel subset of $\Gamma_{0}$. We equip $\Gamma_{\Lambda}$ with the topology induced by that of $\Gamma_{0}$. Let $\mathcal{B}\left(\Gamma_{\Lambda}\right)$ be the corresponding Borel $\sigma$-field. It can be proved, see [26, Lemma 1.1 and Proposition 1.3], that

$$
\mathcal{B}\left(\Gamma_{\Lambda}\right)=\left\{\Gamma_{\Lambda} \cap \Upsilon: \Upsilon \in \mathcal{B}(\Gamma)\right\} .
$$

It is known [1, page 451] that $\mathcal{B}(\Gamma)$ is the smallest $\sigma$-field of subsets of $\Gamma$ such that all the projections

$$
\Gamma \ni \gamma \mapsto p_{\Lambda}(\gamma)=\gamma_{\Lambda}:=\gamma \cap \Lambda, \quad \Lambda \in \mathcal{B}_{\mathrm{b}}\left(\mathbb{R}^{d}\right),
$$

are $\mathcal{B}(\Gamma) / \mathcal{B}\left(\Gamma_{\Lambda}\right)$ measurable. This means that $(\Gamma, \mathcal{B}(\Gamma))$ is the projective limit of the measurable spaces $\left(\Gamma_{\Lambda}, \mathcal{B}\left(\Gamma_{\Lambda}\right)\right), \Lambda \in \mathcal{B}_{\mathrm{b}}\left(\mathbb{R}^{d}\right)$.

Remark 2.1 From the latter discussion it follows that $\Gamma_{0} \in \mathcal{B}(\Gamma)$ and

$$
\mathcal{B}\left(\Gamma_{0}\right)=\left\{A \cap \Gamma_{0}: A \in \mathcal{B}(\Gamma)\right\} .
$$

Hence, a probability measure $\mu$ on $\mathcal{B}(\Gamma)$ with the property $\mu\left(\Gamma_{0}\right)=1$ can be considered also as a measure on $\mathcal{B}\left(\Gamma_{0}\right)$.

\subsection{Functions and Measures on Configuration Spaces}

A Borel set $\Upsilon \subset \Gamma$ is said to be bounded if the following holds

$$
\Upsilon \subset \bigcup_{n=0}^{N} \Gamma_{\Lambda}^{(n)},
$$


for some $\Lambda \in \mathcal{B}_{\mathrm{b}}\left(\mathbb{R}^{d}\right)$ and $N \in \mathbb{N}$. In view of (2.2), each bounded set is in $\mathcal{B}\left(\Gamma_{0}\right)$. A function $G: \Gamma_{0} \rightarrow \mathbb{R}$ is measurable if and only if, for each $n \in \mathbb{N}$, there exists a symmetric Borel function $G^{(n)}:\left(\mathbb{R}^{d}\right)^{n} \rightarrow \mathbb{R}$ such that

$$
G(\eta)=G^{(n)}\left(x_{1}, \ldots, x_{n}\right), \text { for } \eta=\left\{x_{1}, \ldots, x_{n}\right\} .
$$

Definition 2.2 A bounded measurable function $G: \Gamma_{0} \rightarrow \mathbb{R}$ is said to have bounded support if: (a) $G(\eta)=0$ whenever $\eta \cap \Lambda^{c} \neq \emptyset$ for some $\Lambda \in \mathcal{B}_{\mathrm{b}}\left(\mathbb{R}^{d}\right), \Lambda^{c}:=\mathbb{R}^{d} \backslash \Lambda$; (b) $G^{(n)} \equiv 0$ whenever $n>N$ for some $N \in \mathbb{N}$. The set of all such functions is denoted by $B_{\mathrm{bs}}\left(\Gamma_{0}\right)$. For a given $G \in B_{\mathrm{bs}}\left(\Gamma_{0}\right)$, by $N(G)$ we denote the smallest $N$ with the property as in (b).

A map $F: \Gamma \rightarrow \mathbb{R}$ is called cylinder function if there exist $\Lambda \in \mathcal{B}_{\mathrm{b}}\left(\mathbb{R}^{d}\right)$ and a measurable $G: \Gamma_{\Lambda} \rightarrow \mathbb{R}$ such that, cf. $(2.1), F(\gamma)=G\left(\gamma_{\Lambda}\right)$ for all $\gamma \in \Gamma$. Clearly, such a map $F$ is measurable. By $\mathcal{F}_{\text {cyl }}(\Gamma)$ we denote the set of all cylinder functions. For $\gamma \in \Gamma$, by writing $\eta \Subset \gamma$ we mean that $\eta \subset \gamma$ and $\eta$ is finite, i.e., $\eta \in \Gamma_{0}$. For $G \in B_{\mathrm{bs}}\left(\Gamma_{0}\right)$, we set

$$
(K G)(\gamma)=\sum_{\eta \Subset \gamma} G(\eta), \quad \gamma \in \Gamma .
$$

As proved in [17], $K$ maps $B_{\text {bs }}\left(\Gamma_{0}\right)$ onto $\mathcal{F}_{\text {cyl }}(\Gamma)$ and is invertible. The Lebesgue-Poisson measure $\lambda$ on $\mathcal{B}\left(\Gamma_{0}\right)$ is defined by the relation

$$
\int_{\Gamma_{0}} G(\eta) \lambda(d \eta)=G(\emptyset)+\sum_{n=1}^{\infty} \frac{1}{n !} \int_{\left(\mathbb{R}^{d}\right)^{n}} G^{(n)}\left(x_{1}, \ldots, x_{n}\right) d x_{1} \cdots d x_{n},
$$

which has to hold for all $G \in B_{\mathrm{bs}}\left(\Gamma_{0}\right)$, cf. (2.3). Note that $B_{\mathrm{bs}}\left(\Gamma_{0}\right)$ is a measure defining class. Clearly, $\lambda(\Upsilon)<\infty$ for each bounded $\Upsilon \in \mathcal{B}\left(\Gamma_{0}\right)$. With the help of (2.5), we rewrite (1.7) in the following form

$$
B_{\mu}(\theta)=\int_{\Gamma_{0}} k_{\mu}(\eta)\left(\prod_{x \in \eta} \theta(x)\right) \lambda(d \eta) .
$$

In the sequel, by saying that something holds for all $\eta$ we mean that it holds for $\lambda$-almost all $\eta \in \Gamma_{0}$. This relates also to (2.3).

Let $\mathcal{P}(\Gamma)$, resp. $\mathcal{P}\left(\Gamma_{0}\right)$, stand for the set of all probability measures on $\mathcal{B}(\Gamma)$, resp. $\mathcal{B}\left(\Gamma_{0}\right)$. Note that $\mathcal{P}\left(\Gamma_{0}\right)$ can be considered as a subset of $\mathcal{P}(\Gamma)$, see Remark 2.1. For a given $\mu \in \mathcal{P}(\Gamma)$, the projection $\mu^{\Lambda}$ is defined as

$$
\mu^{\Lambda}(A)=\mu\left(p_{\Lambda}^{-1}(A)\right), \quad A \in \mathcal{B}\left(\Gamma_{\Lambda}\right),
$$

where $p_{\Lambda}^{-1}(A):=\left\{\gamma \in \Gamma: p_{\Lambda}(\gamma) \in A\right\}$, see (2.1). The projections of the Lebesgue-Poisson measure $\lambda$ are defined in the same way.

Recall that $\mathcal{P}_{\text {anal }}$ (resp. $\mathcal{P}_{\mathrm{sP}}$ ) denotes the set of all those $\mu \in \mathcal{P}(\Gamma)$ for each of which $B_{\mu}$ defined in (1.6), or (2.6), admits continuation to a function on $L^{1}\left(\mathbb{R}^{d}\right)$ analytic in some neighborhood of zero (resp. exponential type entire function). The elements of $\mathcal{P}_{\mathrm{sP}}$ are called sub-Poissonian states. One can show [17, Proposition 4.14] that for each $\Lambda \in \mathcal{B}_{\mathrm{b}}\left(\mathbb{R}^{d}\right)$ and $\mu \in \mathcal{P}_{\mathrm{sP}}, \mu^{\Lambda}$ is absolutely continuous with respect to $\lambda^{\Lambda}$. The Radon-Nikodym derivative

$$
R_{\mu}^{\Lambda}(\eta)=\frac{d \mu^{\Lambda}}{d \lambda^{\Lambda}}(\eta), \quad \eta \in \Gamma_{\Lambda}
$$


and the correlation function $k_{\mu}$ satisfy

$$
k_{\mu}(\eta)=\int_{\Gamma_{\Lambda}} R_{\mu}^{\Lambda}(\eta \cup \xi) \lambda^{\Lambda}(d \xi), \quad \eta \in \Gamma_{\Lambda},
$$

which holds for all $\Lambda \in \mathcal{B}_{\mathrm{b}}\left(\mathbb{R}^{d}\right)$. Note that (2.9) ties $R_{\mu}^{\Lambda}$ with the restriction of $k_{\mu}$ to $\Gamma_{\Lambda}$. The fact that these are the restrictions of one and the same function $k_{\mu}: \Gamma_{0} \rightarrow \mathbb{R}$ corresponds to the Kolmogorov consistency of the family $\left\{\mu^{\Lambda}\right\}_{\Lambda \in \mathcal{B}_{\mathrm{b}}\left(\mathbb{R}^{d}\right)}$.

By (2.4), (2.7), and (2.9) we get

$$
\int_{\Gamma}(K G)(\gamma) \mu(d \gamma)=\left\langle\left\langle G, k_{\mu}\right\rangle\right\rangle
$$

which holds for each $G \in B_{\mathrm{bs}}\left(\Gamma_{0}\right)$ and $\mu \in \mathcal{P}_{\mathrm{sP}}$. Here and in the sequel we use the notation

$$
\langle\langle G, k\rangle\rangle=\int_{\Gamma_{0}} G(\eta) k(\eta) \lambda(d \eta),
$$

Define

$$
B_{\mathrm{bs}}^{\star}\left(\Gamma_{0}\right)=\left\{G \in B_{\mathrm{bs}}\left(\Gamma_{0}\right): K G \not \equiv 0,(K G)(\gamma) \geq 0 \text { for all } \gamma \in \Gamma\right\} .
$$

By [17, Theorems 6.1 and 6.2 and Remark6.3] we know that the following holds.

Proposition 2.3 Let a measurable function $k: \Gamma_{0} \rightarrow \mathbb{R}$ have the following properties:

(i) $\langle\langle G, k\rangle\rangle \geq 0$ for all $G \in B_{\mathrm{bs}}^{\star}\left(\Gamma_{0}\right)$,

(ii) $k(\emptyset)=1, \quad$ (iii) $k(\eta) \leq C^{|\eta|}, \quad \eta \in \Gamma_{0}$,

property (iii) holding for some $C>0$. Then there exists a unique $\mu \in \mathcal{P}_{\mathrm{SP}}$ for which $k$ is the correlation function.

Finally, we mention the convention

$$
\sum_{a \in \emptyset} \phi_{a}:=0, \quad \prod_{a \in \emptyset} \psi_{a}:=1
$$

which we use in the sequel and the integration rule, see, e.g. [10],

$$
\int_{\Gamma_{0}} \sum_{\xi \subset \eta} H(\xi, \eta \backslash \xi, \eta) \lambda(d \eta)=\int_{\Gamma_{0}} \int_{\Gamma_{0}} H(\xi, \eta, \eta \cup \xi) \lambda(d \xi) \lambda(d \eta),
$$

valid for appropriate functions $H$.

\subsection{Spaces of Functions}

For each $\mu \in \mathcal{P}_{\mathrm{sP}}$, the correlation function satisfies (1.8) in view of which we introduce the following Banach spaces. For $\alpha \in \mathbb{R}$, we set

$$
\|k\|_{\alpha}=\underset{\eta \in \Gamma_{0}}{\operatorname{ess} \sup }|k(\eta)| \exp (-\alpha|\eta|) .
$$

It is a norm that can also be written as follows. As in (2.3), each $k: \Gamma_{0} \rightarrow \mathbb{R}$ is defined by its restrictions to $\Gamma^{(n)}$. Let $k^{(n)}:\left(\mathbb{R}^{d}\right)^{n} \rightarrow \mathbb{R}$ be a symmetric Borel function such that $k^{(n)}\left(x_{1}, \ldots, x_{n}\right)=k(\eta)$ for $\eta=\left\{x_{1}, \ldots, x_{n}\right\}$. We then assume that $k^{(n)} \in L^{\infty}\left(\left(\mathbb{R}^{d}\right)^{n}\right)$, $n \in \mathbb{N}$, cf. (1.8), and define

$$
\|k\|_{\alpha}=\sup _{n \in \mathbb{N}_{0}} e^{-\alpha n} v_{n}(k), \quad v_{n}(k):=\left\|k^{(n)}\right\|_{L^{\infty}\left(\left(\mathbb{R}^{d}\right)^{n}\right)},
$$


that yields the same norm as in (2.14). Obviously,

$$
\mathcal{K}_{\alpha}:=\left\{k: \Gamma_{0} \rightarrow \mathbb{R}:\|k\|_{\alpha}<\infty\right\},
$$

is a Banach space. For $\alpha^{\prime}<\alpha^{\prime \prime}$, we have $\|k\|_{\alpha^{\prime \prime}} \leq\|k\|_{\alpha^{\prime}}$. Hence,

$$
\mathcal{K}_{\alpha^{\prime}} \hookrightarrow \mathcal{K}_{\alpha^{\prime \prime}}, \quad \text { for } \alpha^{\prime}<\alpha^{\prime \prime} .
$$

Here and in the sequel $X \hookrightarrow Y$ denotes continuous embedding. For $\alpha \in \mathbb{R}$, we define, cf. (2.11) and (2.10),

$$
\mathcal{K}_{\alpha}^{\star}=\left\{k \in \mathcal{K}_{\alpha}: \forall G \in B_{\mathrm{bs}}^{\star}\left(\Gamma_{0}\right)\langle\langle G, k\rangle \geq 0\} .\right.
$$

It is a subset of the cone

$$
\mathcal{K}_{\alpha}^{+}=\left\{k \in \mathcal{K}_{\alpha}: k(\eta) \geq 0 \text { for a.a. } \eta \in \Gamma_{0}\right\} .
$$

By Proposition 2.3 we have that each $k \in \mathcal{K}_{\alpha}^{\star}$ with the property $k(\emptyset)=1$ is the correlation function of a unique $\mu \in \mathcal{P}_{\mathrm{sP}}$. We also put

$$
\mathcal{K}_{\infty}=\bigcup_{\alpha \in \mathbb{R}} \mathcal{K}_{\alpha}
$$

and equip this set with the inductive topology.

Finally, we define

$$
\mathcal{K}_{\infty}^{\star}=\bigcup_{\alpha \in \mathbb{R}} \mathcal{K}_{\alpha}^{\star}
$$

\section{The Model and the Results}

\subsection{The Model}

As was already mentioned, the model is specified by the expression given in (1.4). Regarding the kernels in (1.5) we suppose that

$$
a^{ \pm} \in L^{1}\left(\mathbb{R}^{d}\right) \cap L^{\infty}\left(\mathbb{R}^{d}\right), \quad a^{ \pm}(x)=a^{ \pm}(-x) \geq 0,
$$

and thus define

$$
\left\langle a^{ \pm}\right\rangle=\int_{\mathbb{R}^{d}} a^{ \pm}(x) d x, \quad\left\|a^{ \pm}\right\|=\underset{x \in \mathbb{R}^{d}}{\operatorname{ess} \sup } a^{ \pm}(x),
$$

and

$$
E^{ \pm}(\eta)=\sum_{x \in \eta} E^{ \pm}(x, \eta \backslash x)=\sum_{x \in \eta} \sum_{y \in \eta \backslash x} a^{ \pm}(x-y), \quad \eta \in \Gamma_{0} .
$$

We also denote

$$
E(\eta)=\sum_{x \in \eta}\left(m+E^{-}(x, \eta \backslash x)\right)=m|\eta|+E^{-}(\eta),
$$

where $m$ is the same as in (1.4).

In addition to the standard assumptions (3.1) we shall use the following

Assumption $1((b, \vartheta)$-assumption) There exist $\vartheta>0$ and $b \geq 0$ such that the functions introduced in (3.3) satisfy

$$
b|\eta|+E^{-}(\eta) \geq \vartheta E^{+}(\eta), \quad \eta \in \Gamma_{0} .
$$


Note that the case of point-wise domination

$$
a^{-}(x) \geq \vartheta a^{+}(x), \quad x \in \mathbb{R}^{d},
$$

cf. [13, Eq. (3.11)], corresponds to (3.5) with $b=0$. In Sect. 3.4 below we give examples of the kernels $a^{ \pm}$which satisfy (3.5). To exclude the trivial case of $a^{+}=a^{-}=0$ we also assume that

$$
\left\langle a^{-}\right\rangle>0 .
$$

\subsection{The Results}

\subsubsection{The Operators}

In view of the relationship between states and correlation functions discussed in Sect. 2.3, we describe the system's dynamics in the following way. First we obtain the evolution $k_{\mu_{0}} \mapsto k_{t}$ by proving the existence of a unique solution of the Cauchy problem of the following type

$$
\frac{d k_{t}}{d t}=L^{\Delta} k_{t},\left.\quad k_{t}\right|_{t=0}=k_{\mu_{0}},
$$

where the action of $L^{\Delta}$ is calculated from (1.4). Thereafter, we show that each $k_{t}$ has property $k_{t}(\emptyset)=1$ and lies in $\mathcal{K}_{\alpha}^{\star}$ for some $\alpha \in \mathbb{R}$. Hence, it is the correlation function of a unique

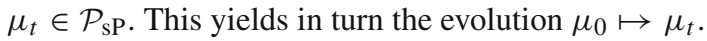

To describe the action of $L^{\Delta}$ in a systematic way we write it in the following form, see $[10,13]$,

$$
L^{\Delta}=A^{\Delta}+B^{\Delta},
$$

where

$$
\begin{aligned}
A^{\Delta} & =A_{1}^{\Delta}+A_{2}^{\Delta}, \\
\left(A_{1}^{\Delta} k\right)(\eta) & =-E(\eta) k(\eta), \\
\left(A_{2}^{\Delta} k\right)(\eta) & =\sum_{x \in \eta} E^{+}(x, \eta \backslash x) k(\eta \backslash x),
\end{aligned}
$$

see also (3.3), (3.4), and

$$
\begin{aligned}
B^{\Delta} & =B_{1}^{\Delta}+B_{2}^{\Delta}, \\
\left(B_{1}^{\Delta} k\right)(\eta) & =-\int_{\mathbb{R}^{d}} E^{-}(y, \eta) k(\eta \cup y) d y, \\
\left(B_{2}^{\Delta} k\right)(\eta) & =\int_{\mathbb{R}^{d}} \sum_{x \in \eta} a^{+}(x-y) k(\eta \backslash x \cup y) d y .
\end{aligned}
$$

The key idea of the method that we use to study (3.7) is to employ the scale of spaces (2.16) in which $A^{\Delta}$ and $B^{\Delta}$ act as bounded operators from $\mathcal{K}_{\alpha^{\prime}}$, to any $\mathcal{K}_{\alpha}$ with $\alpha>\alpha^{\prime}$, cf. (2.17). For such $\alpha$ and $\alpha^{\prime}$, by (2.14) and (2.15) we have, see (3.9),

$$
\begin{aligned}
& \left\|A_{1}^{\Delta} k\right\|_{\alpha} \leq\|k\|_{\alpha^{\prime}} \operatorname{ess} \sup _{\eta \in \Gamma_{0}} E(\eta) \exp \left(-\left(\alpha-\alpha^{\prime}\right)|\eta|\right), \\
& \left\|A_{2}^{\Delta} k\right\|_{\alpha} \leq \underset{\eta \in \Gamma_{0}}{\operatorname{ess} \sup _{0}} e^{-\alpha|\eta|} \sum_{x \in \eta} E^{+}(x, \eta \backslash x)|k(\eta \backslash x)| \\
& \leq\|k\|_{\alpha^{\prime}} e^{-\alpha^{\prime}} \underset{\eta \in \Gamma_{0}}{\operatorname{ess} \sup _{0}} E^{+}(\eta) \exp \left(-\left(\alpha-\alpha^{\prime}\right)|\eta|\right),
\end{aligned}
$$


which by (2.15) and (3.2) yields

$$
\begin{aligned}
\left\|A_{1}^{\Delta} k\right\|_{\alpha} & \leq\|k\|_{\alpha^{\prime}}\left(\frac{m}{e\left(\alpha-\alpha^{\prime}\right)}+\frac{4\left\|a^{-}\right\|}{e^{2}\left(\alpha-\alpha^{\prime}\right)^{2}}\right) \\
\left\|A_{2}^{\Delta} k\right\|_{\alpha} & \leq\|k\|_{\alpha^{\prime}} e^{-\alpha^{\prime}} \frac{4\left\|a^{+}\right\|}{e^{2}\left(\alpha-\alpha^{\prime}\right)^{2}},
\end{aligned}
$$

where we have used the estimate

$$
n^{p} e^{-\sigma n} \leq\left(\frac{p}{e \sigma}\right)^{p}, \quad p \geq 1, \sigma>0, n \in \mathbb{N} .
$$

In a similar way, we obtain from (3.10) the following estimate, see (3.2),

$$
\left\|B^{\Delta} k\right\|_{\alpha} \leq\|k\|_{\alpha^{\prime}} \frac{\left\langle a^{+}\right\rangle+\left\langle a^{-}\right\rangle e^{\alpha^{\prime}}}{e\left(\alpha-\alpha^{\prime}\right)} .
$$

Thus, by means of (3.8) - (3.10), and then by (3.11) and (3.13), for each $\alpha, \alpha^{\prime} \in \mathbb{R}, \alpha^{\prime}<\alpha$, one can define a continuous operator

$$
L_{\alpha \alpha^{\prime}}^{\Delta}: \mathcal{K}_{\alpha^{\prime}} \rightarrow \mathcal{K}_{\alpha}
$$

Let $\mathcal{L}\left(\mathcal{K}_{\alpha^{\prime}}, \mathcal{K}_{\alpha}\right)$ stand for the set of all bounded linear operators $\mathcal{K}_{\alpha^{\prime}} \rightarrow \mathcal{K}_{\alpha}$. The operator norm of $L_{\alpha \alpha^{\prime}}^{\Delta}$ can be estimated by means of the above formulas. Thus, the family $\left\{L_{\alpha \alpha^{\prime}}^{\Delta}\right\}_{\alpha, \alpha^{\prime}}$ determines a continuous linear operator $L^{\Delta}: \mathcal{K}_{\infty} \rightarrow \mathcal{K}_{\infty}$. Along with them in each $\mathcal{K}_{\alpha}$, $\alpha \in \mathbb{R}$, we define an unbounded operator, $L_{\alpha}^{\Delta}$, with domain

$$
\mathcal{D}_{\alpha}^{\Delta}=\left\{k \in \mathcal{K}_{\alpha}: L^{\Delta} k \in \mathcal{K}_{\alpha}\right\} \supset \mathcal{K}_{\alpha^{\prime}},
$$

with the inclusion holding for each $\alpha^{\prime}<\alpha$, see (3.11), (3.13), and (3.8). The operators such introduced are related to each other in the following way:

$$
\forall \alpha^{\prime}<\alpha \quad \forall k \in \mathcal{K}_{\alpha^{\prime}} \quad L_{\alpha \alpha^{\prime}}^{\Delta} k=L_{\alpha}^{\Delta} k .
$$

\subsubsection{The Statements}

Now we can make precise which equations we are going to solve. One possibility is to consider (3.7) in a given Banach space, $\mathcal{K}_{\alpha}$.

Definition 3.1 Given $\alpha \in \mathbb{R}$ and $T \in(0,+\infty)$, by a solution of the Cauchy problem

$$
\frac{d}{d t} k_{t}=L_{\alpha}^{\Delta} k_{t},\left.\quad k_{t}\right|_{t=0}=k_{0} \in \mathcal{D}_{\alpha}^{\Delta},
$$

in $\mathcal{K}_{\alpha}$ we mean a continuous map $[0, T) \ni t \mapsto k_{t} \in \mathcal{D}_{\alpha}$, continuously differentiable in $\mathcal{K}_{\alpha}$ on $[0, T)$ and such that (3.17) is satisfied for all $t \in[0, T)$.

Another possibility is to define (3.7) in the locally convex space (2.20).

Definition 3.2 By a global solution of the Cauchy problem (3.7) in $\mathcal{K}_{\infty}$ with a given $k_{0} \in \mathcal{K}_{\infty}$ we mean a map $[0,+\infty) \ni t \mapsto k_{t} \in \mathcal{K}_{\infty}$, continuously differentiable on $[0,+\infty)$ and such that (3.7) is satisfied for all $t \geq 0$.

According to Definition 3.2, for each $T<+\infty$, there exist $\alpha_{0}, \alpha \in \mathbb{R}, \alpha_{0}<\alpha$, for which the mentioned $k_{t}$ is a solution as in Definition 3.1 with $k_{0} \in \mathcal{K}_{\alpha_{0}}$. Our main results are contained in the following two statements. 
Theorem 3.3 Let $(b, \vartheta)$-assumption (3.5) hold true, and $\mu_{0}$ be an arbitrarily sub-Poissonian state. Then the problem (3.7) with $k_{0}=k_{\mu_{0}}$ has a unique global solution $k_{t} \in \mathcal{K}_{\infty}^{\star}$ with property $k_{t}(\emptyset)=1$. Therefore, for each $t \geq 0$ there exists a unique sub-Poissonian measure $\mu_{t}$ such that $k_{t}=k_{\mu_{t}}$.

The next statement describes the solutions in more detail.

Theorem 3.4 Let $(b, \vartheta)$-assumption (3.5) hold true with $b>0$ (resp. $b=0$ ), and let $\alpha_{0}$ be such that $k_{\mu_{0}} \in \mathcal{K}_{\alpha_{0}}$. Then the solution $k_{t}$ as in Theorem 3.3, corresponding to this $k_{\mu_{0}}$, for all $t \geq 0$, satisfies the following estimates.

(i) Case $\left\langle a^{+}\right\rangle>0$ and $m \in\left[0,\left\langle a^{+}\right\rangle\right]$: for each $\delta<m$ (resp. $\delta \leq m$ ) there exists a positive $C_{\delta}$ such that $\log C_{\delta} \geq \alpha_{0}$ and

$$
k_{t}(\eta) \leq C_{\delta}^{|\eta|} \exp \left[\left(\left\langle a^{+}\right\rangle-\delta\right)|\eta| t\right], \quad \eta \in \Gamma_{0} .
$$

(ii) Case $\left\langle a^{+}\right\rangle>0$ and $m>\left\langle a^{+}\right\rangle$: for each $\varepsilon \in\left(0, m-\left\langle a^{+}\right\rangle\right)$, there exists a positive $C_{\varepsilon}$ such that $\log C_{\varepsilon} \geq \alpha_{0}$ and

$$
k_{t}(\eta) \leq C_{\varepsilon}^{|\eta|} \exp (-\varepsilon t), \quad \eta \neq \emptyset .
$$

(iii) Case $\left\langle a^{+}\right\rangle=0$ :

$$
k_{t}(\eta) \leq k_{0}(\eta) \exp [-E(\eta) t], \quad \eta \in \Gamma_{0} .
$$

If $m=0$ and $a^{-}(x)=\vartheta a^{+}(x)$, then

$$
k_{t}(\eta)=\vartheta^{-|\eta|}, \quad t \geq 0,
$$

is a stationary solution.

Corollary 3.5 In case (i) of Theorem 3.4, for each $T>0, k_{t}$ solves (3.17) in $\mathcal{K}_{\alpha_{T}}$ on the time interval $[0, T)$, where

$$
\alpha_{T}=\log C_{\delta}+\left(\left\langle a^{+}\right\rangle-\delta\right) T .
$$

In case (ii) (resp. (iii)), $k_{t}$ solves (3.17) in $\mathcal{K}_{\alpha}, \alpha=\log C_{\varepsilon}$ (resp. any $\alpha>\alpha_{0}$ ) on the time interval $[0,+\infty)$.

\subsection{Comments and Comparison}

\subsubsection{Comments on the Basic Assumption}

By means of the function

$$
\phi_{\vartheta}(x)=a^{-}(x)-\vartheta a^{+}(x)
$$

one can rewrite (3.5) in the following form

$$
\sum_{x \in \eta} \sum_{y \in \eta \backslash x} \phi_{\vartheta}(x-y) \geq-b|\eta|, \quad \eta \in \Gamma_{0} .
$$

This resembles the stability condition (with stability constant $b \geq 0$ ) for the interaction potential $\phi_{\vartheta}$ used in the statistical mechanics of continuum systems of interacting particles, see [31, Chapter 3]. Below we employ some techniques developed therein to prove that important classes of the kernels $a^{ \pm}$have this property, see Propositions 3.7 and 3.8.

The $(b, \vartheta)$ assumption holds with $b=0$ if and only if (3.6) does. In this case, the dispersal kernel $a^{+}$decays faster than the competition kernel $a^{-}$(short dispersal). It can be 
characterized as the possibility for each daughter-entity to kill her mother-entity, or to be killed by her. In the previous works on this model $[10,12,13]$ the results were based on this short dispersal condition. The novelty of the result of Proposition 3.7 is that it covers also the case of long dispersal where the range of $a^{+}$is finite but can be bigger than that of $a^{-}$. Noteworthy, by our Proposition 3.7 it follows that the interaction potential $\Phi$ used in [29] is stable, which was unknown to the authors of that paper, cf. [29, page 146]. Proposition 3.8 describes Gaussian kernels, for which the basic assumption is valid also for both long and short dispersals. In this paper, we restricted our attention to the classes of kernels described in Propositions 3.7 and 3.8. Extensions beyond this classes, which we plan to realize in a separate work, can be made by means of the corresponding methods of the statistical mechanics of interacting particle systems.

\subsubsection{Comments on the Results}

An important feature of Theorems 3.3 and 3.4 is that the intrinsic mortality rate $m \geq 0$ can be arbitrary. Theorem 3.3 gives a general existence of the evolution $\mu_{0} \mapsto \mu_{t}, t>0$, in the class of sub-Poissonian states through the evolution of the corresponding correlation functions. Its ecological outcome is that the competition in the form as in (1.4), (1.5) excludes clustering provided the kernels satisfy (3.5). A complete characterization of the evolution $k_{0} \mapsto k_{t}$ is then given in Theorem 3.4. By means of it this evolution is 'localized' in the spaces $\mathcal{K}_{\alpha}$ in Corollary 3.5. According to Theorem 3.4, for $m\left\langle\left\langle a^{+}\right\rangle\right.$, or $m \leq\left\langle a^{+}\right\rangle$and $b>0$ in (3.24), the evolution described in Theorem 3.3 takes place in an ascending sequence $\left\{\mathcal{K}_{\alpha_{T}}\right\}_{T \geq 0}$ of Banach spaces, see (2.14) - (2.17), and also (3.22). If $m>\left\langle a^{+}\right\rangle$, the evolution holds in one and the same space, see Corollary 3.5. The only difference between the cases of $b>0$ and $b=0$ is that one can take $\delta=m$ in the latter case. This yields different results for $m=\left\langle a^{+}\right\rangle$, where the evolution takes place in the same space $\mathcal{K}_{\alpha}$ with $\alpha=\log C_{m}$. Note also that for $m=0$, one should take $\delta<0$. For $m>\left\langle a^{+}\right\rangle$, it follows from (3.19) that the population dies out: for $\left\langle a^{+}\right\rangle>0$, the following holds

$$
k_{\mu_{t}}^{(n)}\left(x_{1}, \ldots, x_{n}\right) \leq e^{-\varepsilon t} k_{\mu_{0}}^{(n)}\left(x_{1}, \ldots, x_{n}\right), \quad t>0,
$$

for some $\varepsilon \in\left(0, m-\left\langle a^{+}\right\rangle\right)$, almost all $\left(x_{1}, \ldots, x_{n}\right)$, and each $n \in \mathbb{N}$. For $m>0$ and $\left\langle a^{+}\right\rangle=0$, by (3.20) we get

$$
k_{\mu_{t}}^{(n)}\left(x_{1}, \ldots, x_{n}\right) \leq \exp (-n m t) k_{\mu_{0}}^{(n)}\left(x_{1}, \ldots, x_{n}\right), \quad t>0 .
$$

This means that $k_{\mu_{t}}^{(n)}\left(x_{1}, \ldots, x_{n}\right) \rightarrow 0$ as $n \rightarrow+\infty$ for sufficiently big $t>0$. This phenomenon does not follow from (3.19). Finally, we mention that (3.21) corresponds to a special case of (3.6) and $m=b=0$.

\subsubsection{Comparison}

Here we compare Theorems 3.3 and 3.4 with the corresponding results obtained for this model in $[10,12]$ (where it was called BDLP model), and in [13]. Note that these are the only works where the infinite particle version of the model considered here was studied. In $[10,12]$, the model was supposed to satisfy the conditions, see [12, Eqs. (3.38) and (3.39)], which in the present notations can be formulated as follows: (a) (3.6) holds with a given $\vartheta>0$; (b) $m>16\left\langle a^{-}\right\rangle / \vartheta$ holding with the same $\vartheta$. Under these conditions the global evolution $k_{0} \mapsto k_{t}$ was obtained in $\mathcal{K}_{\alpha}$ with some $\alpha \in \mathbb{R}$ by means of a $C_{0}$-semigroup. No information was available on whether $k_{t}$ is a correlation function and hence on the sign of $k_{t}$. 
In [13], the restrictions were reduced just to (3.6). Then the evolution $k_{0} \mapsto k_{t}$ was obtained in a scale of Banach spaces $\mathcal{K}_{\alpha}$ as in Theorem 3.3, but on a bounded time interval. Like in $[10,12]$, also here no information was obtained on whether $k_{t}$ is a correlation function. Until the present work no results on the extinction as in (3.19) and on the case of $a^{+} \equiv 0$ were known.

\subsection{Kernels Satisfying the Basic Assumption}

Our aim now is to show that the assumption (3.5) can be satisfied in the most of 'realistic' models. We begin, however, by establishing an important property of the kernels satisfying (3.5). To this end we rewrite (3.5) in the form

$$
\Phi_{\vartheta}(\eta):=\sum_{x \in \eta} \sum_{y \in \eta \backslash x}\left[a^{-}(x-y)-\vartheta a^{+}(x-y)\right] \geq-b|\eta|, \quad \eta \in \Gamma_{0} .
$$

Proposition 3.6 Assume that (3.24) holds with some $\vartheta_{0}>0$ and $b_{0} \geq 0$. Then for each $\vartheta<\vartheta_{0}$, it also holds with $b=b_{0} \vartheta / \vartheta_{0}$.

Proof For $\vartheta \in\left(0, \vartheta_{0}\right]$, we have

$$
\Phi_{\vartheta}(\eta)=\frac{\vartheta}{\vartheta_{0}}\left[\left(\frac{\vartheta_{0}}{\vartheta}-1\right) E^{-}(\eta)+\Phi_{\vartheta_{0}}(\eta)\right] \geq-\frac{\vartheta}{\vartheta_{0}} b_{0}|\eta|,
$$

which yields the proof.

In the following two propositions we give examples of the kernels with the property (3.5). In the first one, we assume that the dispersal kernel has finite range, which is quite natural in many applications. The competition kernel in turn is assumed to be just nontrivial.

Proposition 3.7 In addition to (3.1) and (3.2) assume that the kernels $a^{ \pm}$have the following properties:

(a) there exist positive $c^{-}$and $r$ such that $a^{-}(x) \geq c^{-}$for $|x|<r$;

(b) there exist positive $c^{+}$and $R$ such that $a^{+}(x) \leq c^{+}$for $|x|<R$ and $a^{+}(x)=0$ for $|x| \geq R$.

Then for each $b>0$, there exists $\vartheta>0$ such that (3.24) holds for these $b$ and $\vartheta$.

Proof For $r \geq R$, (3.24) holds with $b=0$ and $\vartheta=c^{-} / c^{+}$. Thus, it remains to consider the case $r<R$.

For $|\eta|=0$ and $|\eta|=1$, (3.24) trivially holds with each $b>0$ and $\vartheta>0$. For $|\eta|=2$, (3.24) holds whenever $\vartheta \leq b / c^{+}$. For $|\eta|>2$, we apply an induction in $|\eta|$, similarly as it was done in [2]. For $x \in \eta$, we define

$$
\xi_{x}^{-}=\{y \in \eta:|y-x|<r\}, \quad \xi_{x}^{+}=\{y \in \eta: r \leq|y-x|<R\} .
$$

Set

$$
U_{\vartheta}(\eta)=\Phi_{\vartheta}(\eta)+b|\eta|=b|\eta|+E^{-}(\eta)-\vartheta E^{+}(\eta) .
$$

Then the next estimate holds true for each $x \in \eta$ :

$$
\begin{aligned}
U_{\vartheta}(x, \eta \backslash x) & :=U_{\vartheta}(\eta)-U_{\vartheta}(\eta \backslash x) \\
& =b+2 E^{-}(x, \eta \backslash x)-2 \vartheta E^{+}(x, \eta \backslash x) \\
& \geq b+2\left(c^{-}-\vartheta c^{+}\right)\left|\xi_{x}^{-}\right|-2 \vartheta c^{+}\left|\xi_{x}^{+}\right| .
\end{aligned}
$$


Given $n>2$ and positive $\vartheta$ and $b$, assume that $U_{\vartheta}(\eta) \geq 0$ for each $|\vartheta|=n-1$. Then to make the inductive step by means of (3.25) we have to show that, for each $\eta$ such that $|\eta|=n$, there exists $x \in \eta$ such that $U_{\vartheta}(x, \eta \backslash x) \geq 0$. Set

$$
\bar{n}=\left|\xi_{x}^{-}\right|=\max _{y \in \eta}\left|\xi_{y}^{-}\right|, \quad x \in \eta .
$$

If $\bar{n}=0$, then $\eta$ is such that $|y-z| \geq r$ for each distinct $y, z \in \eta$. In this case, the balls $B_{z}:=\left\{y \in \mathbb{R}^{d}:|y-z|<r / 2\right\}, z \in \eta$, do not overlap. Then $\left|\xi_{x}^{+}\right| \leq \Xi(d, r, R)-1 \leq$ $\Delta(d)(1+2 R / r)^{d}-1$, where $\Xi(d, r, R)$ is the maximum number of rigid spheres of radius $r / 2$ packed in a ball of radius $R+r / 2$, and $\Delta(d)$ is the density of the densest packing of equal rigid spheres in $\mathbb{R}^{d}$, see e.g. [8, Chapter 1]. We apply this in (3.25) and get that $U_{\vartheta}(x, \eta \backslash x) \geq 0$ whenever $\vartheta \leq b / 2 c^{+}(\Xi(d, r, R)-1)$. For $\bar{n}>0$, let $x$ be as in (3.26). Choose $y_{1}, \ldots, y_{s}$ in $\xi_{x}^{+}$such that the balls $B_{x}$ and $B_{y_{i}}, i=1, \ldots, s$, realize the densest possible packing of the ball of radius $R+r / 2$ centered at $x$. Then $s \leq \Xi(d, r, R)-1$ and, for each $y \in \xi_{x}^{+}$, one finds $i$ such that $\left|y-y_{i}\right|<r$. Otherwise $B_{y}$ would not overlap each $B_{y_{i}}$, and thus the mentioned packing is not the densest one. Therefore, the balls $C_{i}:=\left\{z \in \mathbb{R}^{d}:\left|z-y_{i}\right|<r\right\}$, $i=1, \ldots, s$, cover $\xi_{x}^{+}$. By (3.26) each $C_{i}$ contains $\bar{n}+1$ elements at most. This yields

$$
\left|\xi_{x}^{+}\right| \leq(\bar{n}+1)(\Xi(d, r, R)-1) .
$$

Now we apply this in (3.25) and obtain that $U_{\vartheta}(x, \eta \backslash x) \geq 0$ for

$$
\vartheta=\min \left\{\frac{c^{-}}{c^{+} \Xi(d, r, R)} ; \frac{b}{2 c^{+}(\Xi(d, r, R)-1)}\right\} .
$$

Thus, the inductive step can be done, which yields the proof.

As an example of kernels with infinite range we consider the Gaussian kernels

$$
a^{ \pm}(x)=\frac{c_{ \pm}}{\left(2 \pi \sigma_{ \pm}^{2}\right)^{d / 2}} \exp \left(-\frac{1}{2 \sigma_{ \pm}^{2}}|x|^{2}\right),
$$

where $c_{ \pm}>0$ and $\sigma_{ \pm}>0$ are parameters.

Proposition 3.8 Let $a^{ \pm}$be as in (3.27). Then for each $b>0$, there exists $\vartheta$ such that (3.5) holds for these $\vartheta$ and $b$.

Proof For $\sigma_{-} \geq \sigma_{+}$, we have $a^{-}(x) \geq \vartheta a^{+}(x)$ for all $x$ and

$$
\vartheta \leq\left(\frac{\sigma_{+} c_{-}^{1 / d}}{\sigma_{-} c_{+}^{1 / d}}\right)^{d} \text {. }
$$

Then (3.24), and thus (3.5), hold for such $\vartheta$ and all $b \geq 0$. For $\sigma_{-}<\sigma_{+}$, we can write, see (3.23),

$$
\phi_{\vartheta}(x)=\int_{\mathbb{R}^{d}} \hat{\phi}_{\vartheta}(k) \exp (i k \cdot x) d k
$$

where

$$
\hat{\phi}_{\vartheta}(k)=c_{-} \exp \left(-\frac{1}{2} \sigma_{-}^{2}|k|^{2}\right)\left[1-\vartheta \frac{c_{+}}{c_{-}} \exp \left(-\frac{1}{2}\left(\sigma_{+}^{2}-\sigma_{-}^{2}\right)|k|^{2}\right)\right]
$$

For $\vartheta_{0}=c_{-} / c_{+}$, we have that $\hat{\phi}_{\vartheta_{0}}(k) \geq 0$ for all $k \in \mathbb{R}^{d}$. Then $\phi_{\vartheta_{0}}$ is positive definite in the sense of [31, Sect. 3.2]. This means that it is the Fourier transform of a positive finite measure on $\mathbb{R}^{d}$, and hence by the Bochner theorem it follows that 


$$
\sum_{x, y \in \eta} \phi_{\vartheta_{0}}(x-y)=\phi_{\vartheta_{0}}(0)|\eta|+\Phi_{\vartheta_{0}}(\eta) \geq 0 .
$$

Thus, $\Phi_{\vartheta_{0}}$ satisfies (3.24) with stability constant $b_{0}=\phi_{\vartheta_{0}}(0)$. Then we apply Proposition 3.6 and obtain that (3.24) holds for

$$
\vartheta=\frac{\left(2 \pi \sigma_{-}^{2}\right)^{d / 2} b}{\sigma_{+}\left(1-\left(\frac{\sigma_{-}}{\sigma_{+}}\right)^{d}\right)}
$$

which completes the proof.

\section{Evolution of Correlation Functions and States}

Our proof of Theorems 3.3 and 3.4 may roughly be divided into the following three steps. First, we show that for each $k_{0} \in \mathcal{K}_{\alpha_{1}}$ and any $\alpha_{2}>\alpha_{1}$, cf. (2.17), the problem in (3.17) has a unique solution in $\mathcal{K}_{\alpha_{2}}$ on the time interval [0,T( $\left.\alpha_{2}, \alpha_{1}\right)$ ) with an explicitly computed $T\left(\alpha_{2}, \alpha_{1}\right)<\infty$, see Lemma 4.8. To this end, in Lemma 4.5 we construct a family of bounded operators, indexed by $t \in\left[0, T\left(\alpha_{2}, \alpha_{1}\right)\right)$ and acting from $\mathcal{K}_{\alpha_{1}}$ to $\mathcal{K}_{\alpha_{2}}$, which gives the solution in question in the way resembling the action of a $C_{0}$-semigroup. Here we employ a combination of the usual Ovcynnikov method, as in e.g. [5], based on the estimates in (4.18) and (4.19), and a substochastic semigroup constructed in the pre-dual space in Lemma 4.2. The construction employs Assumption 1 and a perturbation result of [32] applied to the operator pre-dual to $A_{b}^{\Delta}$ given in (4.15). In this way, we avoid the consequences of the righthand sides of (3.11) related to $\left(\alpha-\alpha^{\prime}\right)^{2}$, bad for using Ovcynnikov's method. However, due to the term $e^{\alpha_{2}}$ in (4.20) the length of the time interval $T\left(\alpha_{2}, \alpha_{1}\right)$ ) is bounded by some $\tau\left(\alpha_{1}\right)<\infty$. This and the fact that $\tau\left(\alpha_{1}\right) \rightarrow 0$ as $\alpha_{1} \rightarrow+\infty$ do not allow one to increase $\left.T\left(\alpha_{2}, \alpha_{1}\right)\right)$ ad infinitum just by increasing the space $\mathcal{K}_{\alpha_{2}}$ containing the solution. To overcome this difficulty, and thus to construct the global solution, we make another two steps. In Lemma 4.9 , we show that the constructed solution $k_{t}$ lies in the cone defined in (2.18), and hence is the correlation function of a unique state $\mu_{t}$, see Proposition 2.3. The relevance of this fact is twofold. First of all, it implies that the evolution $k_{\mu_{0}} \mapsto k_{t}$ corresponds to the uniquely determined evolution of states - the main aim of this work. At the same time, by Lemma 4.9 we obtain that $k_{t}(\eta) \geq 0$. By the comparison made in Lemma 4.10 based on this positivity we get rid of the mentioned term $e^{\alpha_{2}}$, cf. the second line in (4.20). This finally allows us to continue the solution $k_{t}$ to all $t>0$ - the third step - and thereby to construct the solution as claimed in Theorem 3.3. The estimates as in Theorem 3.4 are obtained by the mentioned comparison.

We begin by constructing auxiliary semigroups used to make (in Sect. 4.2) the first step of the construction outlined above.

\subsection{Auxiliary Semigroups}

For a given $\alpha \in \mathbb{R}$, the space predual to $\mathcal{K}_{\alpha}$, defined in (2.16), is

$$
\mathcal{G}_{\alpha}:=L^{1}\left(\Gamma_{0}, e^{\alpha|\cdot|} d \lambda\right)
$$

in which the norm is, cf. (2.5),

$$
\begin{gathered}
|G|_{\alpha}=\int_{\Gamma_{0}}|G(\eta)| \exp (\alpha|\eta|) \lambda(d \eta) \\
=\sum_{n=0}^{\infty} \frac{e^{\alpha n}}{n !}\left\|G^{(n)}\right\|_{L^{1}\left(\left(\mathbb{R}^{d}\right)^{n}\right)} .
\end{gathered}
$$


Clearly, $|G|_{\alpha^{\prime}} \leq|G|_{\alpha}$ for $\alpha^{\prime}<\alpha$, which yields

$$
\mathcal{G}_{\alpha} \hookrightarrow \mathcal{G}_{\alpha^{\prime}}, \quad \text { for } \alpha^{\prime}<\alpha,
$$

cf. (2.17). One can show that this embedding is also dense.

Recall that by $m \geq 0$ we denote the mortality rate, see (1.4). For $b \geq 0$ as in (3.5) we set

$$
E_{b}(\eta)=(b+m)|\eta|+E^{-}(\eta)=b|\eta|+E(\eta) .
$$

Here $E^{-}(\eta)$ and $E(\eta)$ are as in (3.3) and (3.4), respectively. For the same $b$, let the action of $A_{b}$ on functions $G: \Gamma_{0} \rightarrow \mathbb{R}$ be as follows

$$
\begin{aligned}
A_{b} & =A_{1, b}+A_{2} \\
\left(A_{1, b} G\right)(\eta) & =-E_{b}(\eta) G(\eta), \\
\left(A_{2} G\right)(\eta) & =\int_{\mathbb{R}^{d}} E^{+}(y, \eta) G(\eta \cup y) d y .
\end{aligned}
$$

Our aim now is to define $A_{b}$ as a closed unbounded operator in $\mathcal{G}_{\alpha}$ the domain of which contains $\mathcal{G}_{\alpha^{\prime}}$ for any $\alpha^{\prime}>\alpha$. Let $\mathcal{G}_{\alpha}^{+}$denote the set of all those $G \in \mathcal{G}_{\alpha}$ for which $G(\eta) \geq 0$ for $\lambda$-almost all $\eta \in \Gamma_{0}$. Set

$$
\mathcal{D}_{\alpha}=\left\{G \in \mathcal{G}_{\alpha}: E_{b}(\cdot) G(\cdot) \in \mathcal{G}_{\alpha}\right\} .
$$

For each $\alpha^{\prime}>\alpha, \mathcal{D}_{\alpha}$ contains $\mathcal{G}_{\alpha^{\prime}}$ and hence is dense in $\mathcal{G}_{\alpha}$, see (4.3). Then the first summand in $A_{b}$ turns into a closed and densely defined operator $\left(A_{1, b}, \mathcal{D}_{\alpha}\right)$ in $\mathcal{G}_{\alpha}$ such that $-A_{1, b} G \in \mathcal{G}_{\alpha}^{+}$ for each $G \in \mathcal{D}_{\alpha}^{+}:=\mathcal{D}_{\alpha} \cap \mathcal{G}_{\alpha}^{+}$. By (2.13) and (3.5) one gets

$$
\begin{aligned}
\left|A_{2} G\right|_{\alpha} & \leq \int_{\Gamma_{0}} \int_{\mathbb{R}^{d}} E^{+}(y, \eta)|G(\eta \cup y)| e^{\alpha|\eta|} d y \lambda(d \eta) \\
& =e^{-\alpha} \int_{\Gamma_{0}}|G(\eta)| e^{\alpha|\eta|}\left(\sum_{x \in \eta} E^{+}(x, \eta \backslash x)\right) \lambda(d \eta) \\
& =e^{-\alpha}\left|E^{+}(\cdot) G(\cdot)\right|_{\alpha} \leq\left(e^{-\alpha} / \vartheta\right)\left|A_{1, b} G\right|_{\alpha} .
\end{aligned}
$$

Then for $\alpha>-\log \vartheta$, we have that $e^{-\alpha} / \vartheta<1$, and hence $A_{2}$ is $A_{1, b}$-bounded. This means that $\left(A_{b}, \mathcal{D}_{\alpha}\right)$ is closed and densely defined in $\mathcal{G}_{\alpha}$, see (4.5).

In the proof of Lemma 4.2 below we employ the perturbation theory for positive semigroups of operators in ordered Banach spaces developed in [32]. Prior to stating the lemma we present the relevant fragments of this theory in spaces of integrable functions. Let $E$ be a measurable space with a $\sigma$-finite measure $\nu$, and $X:=L^{1}(E \rightarrow \mathbb{R}, d \nu)$ be the Banach space of $\nu$-integrable real-valued functions on $X$ with norm $\|\cdot\|$. Let $X^{+}$be the cone in $X$ consisting of all $v$-a.e. nonnegative functions on $E$. Clearly, $\|f+g\|=\|f\|+\|g\|$ for any $f, g \in X^{+}$, and $X=X^{+}-X^{+}$. Recall that a $C_{0}$-semigroup $\{S(t)\}_{t \geq 0}$ of bounded linear operators on $X$ is called positive if $S(t) f \in X^{+}$for all $f \in X^{+}$. A positive semigroup is called substochastic (resp. stochastic) if $\|S(t) f\| \leq\|f\|$ (resp. $\|S(t) f\|=\|f\|$ ) for all $f \in X^{+}$. Let $\left(A_{0}, D\left(A_{0}\right)\right)$ be the generator of a positive $C_{0}$-semigroup $\left\{S_{0}(t)\right\}_{t \geq 0}$ on $X$. Set $D^{+}\left(A_{0}\right)=D\left(A_{0}\right) \cap X^{+}$. Then $D\left(A_{0}\right)$ is dense in $X$, and $D^{+}\left(A_{0}\right)$ is dense in $X^{+}$. Let $P: D\left(A_{0}\right) \rightarrow X$ be a positive linear operator, i.e., $P f \in X^{+}$for all $f \in D^{+}\left(A_{0}\right)$. The next statement is an adaptation of Theorem 2.2 in [32].

Proposition 4.1 Suppose that for any $f \in D^{+}\left(A_{0}\right)$, the following holds

$$
\int_{E}\left(\left(A_{0}+P\right) f\right)(x) v(d x) \leq 0 .
$$


Then for all $r \in[0,1)$, the operator $\left(A_{0}+r P, D\left(A_{0}\right)\right)$ is the generator of a substochastic $C_{0}$-semigroup in $X$.

Lemma 4.2 For each $\alpha>-\log \vartheta$, the operator $\left(A_{b}, \mathcal{D}_{\alpha}\right)$ is the generator of a substochastic semigroup $\{S(t)\}_{t \geq 0}$ in $\mathcal{G}_{\alpha}$.

Proof We apply Proposition 4.1 with $E=\Gamma_{0}, X=\mathcal{G}_{\alpha}$ as in (4.1), and $A_{0}=A_{1, b}$. For $r>0$ and $A_{2}$ as in (4.5), we set $P=r^{-1} A_{2}$. For such $A_{0}$ and $P$, and for $G \in \mathcal{D}_{\alpha}^{+}$, the left-hand side of (4.8) takes the form, cf. (4.7),

$$
\begin{aligned}
& -\int_{\Gamma_{0}} E_{b}(\eta) G(\eta) \exp (\alpha|\eta|) \lambda(d \eta) \\
& +r^{-1} \int_{\Gamma_{0}} \int_{\mathbb{R}^{d}} E^{+}(y, \eta) G(\eta \cup y) \exp (\alpha|\eta|) d y \lambda(d \eta) \\
& =\int_{\Gamma_{0}}\left(-E_{b}(\eta)+r^{-1} e^{-\alpha} E^{+}(\eta)\right) G(\eta) \exp (\alpha|\eta|) \lambda(d \eta) .
\end{aligned}
$$

For a fixed $\alpha>-\log \vartheta$, pick $r \in(0,1)$ such that $r^{-1}\left(e^{-\alpha} / \vartheta\right)<1$. Then, for such $\alpha$ and $r$, we have

$$
\int_{\Gamma_{0}}\left(-E_{b}(\eta)+r^{-1} e^{-\alpha} E^{+}(\eta)\right) G(\eta) \exp (\alpha|\eta|) \lambda(d \eta) \leq 0,
$$

which holds in view of (3.5). Since $r^{-1} A_{2}$ is a positive operator, by Proposition 4.1 we have that $A_{b}=A_{1, b}+A_{2}=A_{1, b}+r\left(r^{-1} A_{2}\right)$ generates a substochastic semigroup $\{S(t)\}_{t \geq 0}$ in $\mathcal{G}_{\alpha}$.

Now we turn to constructing the semigroup 'sun-dual' to that mentioned in Lemma 4.2. Let $A_{b}^{*}$ be the adjoint of $\left(A_{b}, \mathcal{D}_{\alpha}\right)$ in $\mathcal{K}_{\alpha}$ with domain, cf. (3.13),

$$
\operatorname{Dom}\left(A_{b}^{*}\right)=\left\{k \in \mathcal{K}_{\alpha}: \exists \tilde{k} \in \mathcal{K}_{\alpha} \forall G \in \mathcal{D}_{\alpha}\left\langle\left\langle A_{b} G, k\right\rangle\right\rangle=\langle\langle G, \tilde{k}\rangle\rangle\right\} .
$$

For each $k \in \operatorname{Dom}\left(A_{b}^{*}\right)$, the action of $A_{b}^{*}$ on $k$ is described in (3.9) with $E$ replaced by $E_{b}$, see (4.4). By (3.11) we then get $\mathcal{K}_{\alpha^{\prime}} \subset \operatorname{Dom}\left(A_{b}^{*}\right)$ for each $\alpha^{\prime}<\alpha$. Let $\mathcal{Q}_{\alpha}$ stand for the closure of $\operatorname{Dom}\left(A_{b}^{*}\right)$ in $\|\cdot\|_{\alpha}$. Then

$$
\mathcal{Q}_{\alpha}:=\overline{\operatorname{Dom}\left(A_{b}^{*}\right)} \supset \operatorname{Dom}\left(A_{b}^{*}\right) \supset \mathcal{K}_{\alpha^{\prime}}, \quad \text { for any } \alpha^{\prime}<\alpha .
$$

Note that $\mathcal{Q}_{\alpha}$ is a proper subset of $\mathcal{K}_{\alpha}$. For each $t \geq 0$, the adjoint $S^{*}(t)$ of $S(t)$ is a bounded operator in $\mathcal{K}_{\alpha}$. However, the semigroup $\left\{S^{*}(t)\right\}_{t \geq 0}$ is not strongly continuous. For $t>0$, let $S_{\alpha}^{\odot}(t)$ denote the restriction of $S^{*}(t)$ to $\mathcal{Q}_{\alpha}$. Since $\{S(t)\}_{t \geq 0}$ is the semigroup of contractions, for $k \in \mathcal{Q}_{\alpha}$ and all $t \geq 0$, we have that

$$
\left\|S_{\alpha}^{\odot}(t) k\right\|_{\alpha}=\left\|S^{*}(t) k\right\|_{\alpha} \leq\|k\|_{\alpha} .
$$

Proposition 4.3 For every $\alpha^{\prime}<\alpha$ and any $k \in \mathcal{K}_{\alpha^{\prime}}$, the map

$$
[0,+\infty) \ni t \mapsto S_{\alpha}^{\odot}(t) k \in \mathcal{K}_{\alpha}
$$

is continuous. 
Proof By [28, Theorem 10.4, page 39], the collection $\left\{S_{\alpha}^{\odot}(t)\right\}_{t \geq 0}$ constitutes a $C_{0}$-semigroup on $\mathcal{Q}_{\alpha}$ the generator of which, $A_{\alpha}^{\odot}$, is the part of $A_{b}^{*}$ in $\mathcal{Q}_{\alpha}$. That is, $A_{\alpha}^{\odot}$ is the restriction of $A_{b}^{*}$ to the set

$$
\operatorname{Dom}\left(A_{\alpha}^{\odot}\right):=\left\{k \in \operatorname{Dom}\left(A_{b}^{*}\right): A_{b}^{*} k \in \mathcal{Q}_{\alpha}\right\},
$$

cf. [28, Definition 10.3, page39]. The continuity in question follows by the $C_{0}$-property of the semigroup $\left\{S_{\alpha}^{\odot}(t)\right\}_{t \geq 0}$ and (4.10).

By (3.11) it follows that

$$
\operatorname{Dom}\left(A_{\alpha^{\prime \prime}}^{\odot}\right) \supset \mathcal{K}_{\alpha^{\prime}}, \quad \alpha^{\prime}<\alpha^{\prime \prime},
$$

and hence, see [28, Theorem 2.4, page4],

$$
S_{\alpha^{\prime \prime}}^{\odot}(t) k \in \operatorname{Dom}\left(A_{\alpha^{\prime \prime}}^{\odot}\right),
$$

and

$$
\frac{d}{d t} S_{\alpha^{\prime \prime}}^{\odot}(t) k=A_{\alpha^{\prime \prime}}^{\odot} S_{\alpha^{\prime \prime}}^{\odot}(t) k,
$$

which holds for all $\alpha^{\prime \prime} \in\left(\alpha^{\prime}, \alpha\right]$ and $k \in \mathcal{K}_{\alpha^{\prime}}$.

\subsection{The Main Operators}

For $E_{b}$ as in (4.4), we set

$$
\begin{aligned}
A_{b}^{\Delta} & =A_{1, b}^{\Delta}+A_{2}^{\Delta}, \\
\left(A_{1, b}^{\Delta} k\right)(\eta) & =-E_{b}(\eta) k(\eta),
\end{aligned}
$$

and $A_{2}^{\Delta}$ being as in (3.9). We also set

$$
\begin{aligned}
B_{b}^{\Delta} & =B_{1}^{\Delta}+B_{2, b}^{\Delta}, \\
\left(B_{2, b}^{\Delta} k\right)(\eta) & =\left(B_{2}^{\Delta} k\right)(\eta)+b|\eta| k(\eta) .
\end{aligned}
$$

Here $B_{1}^{\Delta}$ and $B_{2}^{\Delta}$ are as in (3.10). Note that

$$
L^{\Delta}=A^{\Delta}+B^{\Delta}=A_{b}^{\Delta}+B_{b}^{\Delta} .
$$

The expressions in (4.15) and (4.16) can be used to define the corresponding continuous operators acting from $\mathcal{K}_{\alpha^{\prime}}$ to $\mathcal{K}_{\alpha}, \alpha^{\prime}<\alpha$, cf. (3.14), and hence the elements of $\mathcal{L}\left(\mathcal{K}_{\alpha^{\prime}}, \mathcal{K}_{\alpha}\right)$ the norms of which are estimated by means of the analogies of (3.11) and (3.13). For these operators, we use notations $\left(B_{b}^{\Delta}\right)_{\alpha \alpha^{\prime}}$ and $\left(B_{2, b}^{\Delta}\right)_{\alpha \alpha^{\prime}}$. Then $\left\|\left(B_{b}^{\Delta}\right)_{\alpha \alpha^{\prime}}\right\|$ will stand for the operator norm, and thus (3.13) can be rewritten in the form

$$
\left\|\left(B_{b}^{\Delta}\right)_{\alpha \alpha^{\prime}}\right\| \leq \frac{\left\langle a^{+}\right\rangle+b+\left\langle a^{-}\right\rangle e^{\alpha^{\prime}}}{e\left(\alpha-\alpha^{\prime}\right)} .
$$

For fixed $\alpha>\alpha^{\prime}>-\log \vartheta$, we construct continuous operators $Q_{\alpha \alpha^{\prime}}(t ; \mathbb{B}): \mathcal{K}_{\alpha^{\prime}} \rightarrow \mathcal{K}_{\alpha}$, $t>0$, which will be used to obtain the solution $k_{t}$ as in Theorem 3.3 and to study its properties. Here $\mathbb{B}$ will be taken in the following two versions: (a) $\mathbb{B}=B_{b}^{\Delta}$; (b) $\mathbb{B}=B_{2, b}^{\Delta}$, see (4.16). In both cases, for each $\alpha_{1}, \alpha_{2} \in\left[\alpha^{\prime}, \alpha\right]$ such that $\alpha_{1}<\alpha_{2}$, cf. (4.18), the following holds

$$
\left\|\mathbb{B}_{\alpha_{2} \alpha_{1}}\right\| \leq \frac{\beta\left(\alpha_{2} ; \mathbb{B}\right)}{e\left(\alpha_{2}-\alpha_{1}\right)},
$$


with

$$
\begin{aligned}
& \beta\left(\alpha_{2} ; B_{b}^{\Delta}\right)=\left\langle a^{+}\right\rangle+b+\left\langle a^{-}\right\rangle e^{\alpha_{2}}, \\
& \beta\left(\alpha_{2} ; B_{2, b}^{\Delta}\right)=\left\langle a^{+}\right\rangle+b .
\end{aligned}
$$

For $t>0$ and $\alpha_{1}, \alpha_{2}$ as above, let $\Sigma_{\alpha_{2} \alpha_{1}}(t): \mathcal{K}_{\alpha_{1}} \rightarrow \mathcal{K}_{\alpha_{2}}$ be the restriction of $S_{\alpha_{2}}^{\odot}(t)$ to $\mathcal{K}_{\alpha_{1}}$, cf. (4.12) and (4.13). Note that the embedding $\mathcal{K}_{\alpha_{1}} \hookrightarrow \mathcal{K}_{\alpha_{2}}$. can be written as $\Sigma_{\alpha_{2} \alpha_{1}}(0)$, and hence

$$
\Sigma_{\alpha_{2} \alpha_{1}}(t)=\Sigma_{\alpha_{2} \alpha_{1}}(0) S_{\alpha_{1}}^{\odot}(t) .
$$

Also, for each $\alpha_{3}>\alpha_{2}$, we have

$$
\Sigma_{\alpha_{3} \alpha_{1}}(t)=\Sigma_{\alpha_{3} \alpha_{2}}(0) \Sigma_{\alpha_{2} \alpha_{1}}(t):=\Sigma_{\alpha_{2} \alpha_{1}}(t), \quad t \geq 0 .
$$

Here and in the sequel, we omit writing embedding operators if no confusing arises. In view of (4.11), it follows that

$$
\left\|\Sigma_{\alpha_{2} \alpha_{1}}(t)\right\| \leq 1
$$

Remark 4.4 By Lemma 4.2 we have that

$$
\forall k \in \mathcal{K}_{\alpha_{1}}^{+} \quad \Sigma_{\alpha_{2} \alpha_{1}}(t) k \in \mathcal{K}_{\alpha_{2}}^{+}, \quad t \geq 0,
$$

see (2.19). Also $\left(B_{2, b}^{\Delta}\right)_{\alpha_{2} \alpha_{1}}$, but not $\left(B_{b}^{\Delta}\right)_{\alpha_{2} \alpha_{1}}$, has the same positivity property.

Set, cf. (4.20),

$$
T\left(\alpha_{2}, \alpha_{1} ; \mathbb{B}\right)=\frac{\alpha_{2}-\alpha_{1}}{\beta\left(\alpha_{2} ; \mathbb{B}\right)}, \quad \alpha_{2}>\alpha_{1},
$$

and then

$$
\mathcal{A}(\mathbb{B})=\left\{\left(\alpha_{1}, \alpha_{2}, t\right):-\log \vartheta<\alpha_{1}<\alpha_{2}, \quad 0 \leq t<T\left(\alpha_{2}, \alpha_{1} ; \mathbb{B}\right)\right\} .
$$

Lemma 4.5 For each of the two choices of $\mathbb{B}$, see (4.20), there exists the corresponding family of linear maps, $\left\{Q_{\alpha_{2} \alpha_{1}}(t ; \mathbb{B}):\left(\alpha_{1}, \alpha_{2}, t\right) \in \mathcal{A}(\mathbb{B})\right\}$, each element of which has the following properties:

(i) $Q_{\alpha_{2} \alpha_{1}}(t ; \mathbb{B}) \in \mathcal{L}\left(\mathcal{K}_{\alpha_{1}}, \mathcal{K}_{\alpha_{2}}\right)$;

(ii) the map $\left[0, T\left(\alpha_{2}, \alpha_{1} ; \mathbb{B}\right)\right) \ni t \mapsto Q_{\alpha_{2} \alpha_{1}}(t ; \mathbb{B}) \in \mathcal{L}\left(\mathcal{K}_{\alpha_{1}}, \mathcal{K}_{\alpha_{2}}\right)$ is continuous;

(iii) the operator norm of $Q_{\alpha_{2} \alpha_{1}}(t ; \mathbb{B}) \in \mathcal{L}\left(\mathcal{K}_{\alpha_{1}}, \mathcal{K}_{\alpha_{2}}\right)$ satisfies

$$
\left\|Q_{\alpha_{2} \alpha_{1}}(t ; \mathbb{B})\right\| \leq \frac{T\left(\alpha_{2}, \alpha_{1} ; \mathbb{B}\right)}{T\left(\alpha_{2}, \alpha_{1} ; \mathbb{B}\right)-t} ;
$$

(iv) for each $\alpha_{3} \in\left(\alpha_{1}, \alpha_{2}\right)$ and $t<T\left(\alpha_{3}, \alpha_{1} ; \mathbb{B}\right)$, the following holds

$$
\frac{d}{d t} Q_{\alpha_{2} \alpha_{1}}(t ; \mathbb{B})=\left(\left(A_{b}^{\Delta}\right)_{\alpha_{2} \alpha_{3}}+\mathbb{B}_{\alpha_{2} \alpha_{3}}\right) Q_{\alpha_{3} \alpha_{1}}(t ; \mathbb{B}) .
$$

The proof of this lemma is based on the following construction. For $l \in \mathbb{N}$ and $t>0$, we set

$$
\mathcal{T}_{l}:=\left\{\left(t, t_{1}, \ldots, t_{l}\right): 0 \leq t_{l} \leq \cdots \leq t_{1} \leq t\right\},
$$


take $\alpha \in\left(\alpha_{1}, \alpha_{2}\right]$, and then take $\delta<\alpha-\alpha_{1}$. Next we divide the interval $\left[\alpha_{1}, \alpha\right]$ into subintervals with endpoints $\alpha^{s}, s=0, \ldots, 2 l+1$, as follows. Set $\alpha^{0}=\alpha_{1}, \alpha^{2 l+1}=\alpha$, and

$$
\begin{aligned}
\alpha^{2 s} & =\alpha_{1}+\frac{s}{l+1} \delta+s \epsilon, & \epsilon & =\left(\alpha-\alpha_{1}-\delta\right) / l, \\
\alpha^{2 s+1} & =\alpha_{1}+\frac{s+1}{l+1} \delta+s \epsilon, & s & =0,1, \ldots, l .
\end{aligned}
$$

Then for $\left(t, t_{1}, \ldots, t_{l}\right) \in \mathcal{T}_{l}$, define

$$
\begin{aligned}
& \Pi_{\alpha \alpha_{1}}^{(l)}\left(t, t_{1}, \ldots, t_{l} ; \mathbb{B}\right)=\Sigma_{\alpha \alpha^{2 l}}\left(t-t_{1}\right) \mathbb{B}_{\alpha^{2 l} \alpha^{2 l-1}} \times \cdots \\
& \quad \times \Sigma_{\alpha^{2 s+1} \alpha^{2 s}}\left(t_{l-s}-t_{l-s+1}\right) \mathbb{B}_{\alpha^{2 s} \alpha^{2 s-1}} \cdots \Sigma_{\alpha^{3} \alpha^{2}}\left(t_{l-1}-t_{l}\right) \mathbb{B}_{\alpha^{2} \alpha^{1}} \Sigma_{\alpha^{1} \alpha_{1}}\left(t_{l}\right) .
\end{aligned}
$$

Proposition 4.6 For both choices of $\mathbb{B}$ and each $l \in \mathbb{N}$, the operators defined in (4.30) have the following properties:

(i) for each $\left(t, t_{1}, \ldots, t_{l}\right) \in \mathcal{T}_{l}, \Pi_{\alpha \alpha_{1}}^{(l)}\left(t, t_{1}, \ldots, t_{l} ; \mathbb{B}\right) \in \mathcal{L}\left(\mathcal{K}_{\alpha_{1}}, \mathcal{K}_{\alpha}\right)$, and the map

$$
\mathcal{T}_{l} \ni\left(t, t_{1}, \ldots, t_{l}\right) \mapsto \Pi_{\alpha \alpha_{1}}^{(l)}\left(t, t_{1}, \ldots, t_{l} ; \mathbb{B}\right) \in \mathcal{L}\left(\mathcal{K}_{\alpha_{1}}, \mathcal{K}_{\alpha}\right)
$$

is continuous;

(ii) for fixed $t_{1}, t_{2}, \ldots, t_{l}$, and each $\varepsilon>0$, the map

$$
\left(t_{1}, t_{1}+\varepsilon\right) \ni t \mapsto \Pi_{\alpha \alpha_{1}}^{(l)}\left(t, t_{1}, \ldots, t_{l} ; \mathbb{B}\right) \in \mathcal{L}\left(\mathcal{K}_{\alpha_{1}}, \mathcal{K}_{\alpha_{2}}\right)
$$

is continuously differentiable and for each $\alpha^{\prime} \in\left(\alpha_{1}, \alpha\right)$ the following holds

$$
\frac{d}{d t} \Pi_{\alpha \alpha_{1}}^{(l)}\left(t, t_{1}, \ldots, t_{l} ; \mathbb{B}\right)=\left(A_{b}^{\Delta}\right)_{\alpha \alpha^{\prime}} \Pi_{\alpha^{\prime} \alpha_{1}}^{(l)}\left(t, t_{1}, \ldots, t_{l} ; \mathbb{B}\right)
$$

Proof The first part of claim ( $i$ ) follows by (4.30), (4.19), and (4.23). To prove the second part we apply Proposition 4.3 and (4.21), and then (4.19), (4.20). By (4.12), (4.14), and (4.22), and the fact that

$$
A_{\alpha^{\prime}}^{\odot} k=\left(A_{b}^{\Delta}\right)_{\alpha^{\prime} \alpha} k, \quad \text { for } k \in \mathcal{K}_{\alpha},
$$

one gets

$$
\frac{d}{d t} \Sigma_{\alpha^{\prime} \alpha_{2 l}}(t)=\left(A_{b}^{\Delta}\right)_{\alpha^{\prime} \alpha} \Sigma_{\alpha \alpha_{2 l}}(t), \quad \alpha^{\prime}>\alpha,
$$

which then yields (4.31).

Proof of Lemma 4.5 Take any $T<T\left(\alpha_{2}, \alpha_{1} ; \mathbb{B}\right)$ and then pick $\alpha \in\left(\alpha_{1}, \alpha_{2}\right]$ and a positive $\delta<\alpha-\alpha_{1}$ such that

$$
T<T_{\delta}:=\frac{\alpha-\alpha_{1}-\delta}{\beta\left(\alpha_{2} ; \mathbb{B}\right)} .
$$

For this $\delta$, take $\Pi_{\alpha \alpha_{1}}^{(l)}$ as in (4.30), and then for set

$$
\begin{aligned}
& Q_{\alpha \alpha_{1}}^{(n)}(t ; \mathbb{B})=\Sigma_{\alpha \alpha_{1}}(t) \\
& +\sum_{l=1}^{n} \int_{0}^{t} \int_{0}^{t_{1}} \cdots \int_{0}^{t_{l-1}} \Pi_{\alpha \alpha_{1}}^{(l)}\left(t, t_{1}, \ldots, t_{l} ; \mathbb{B}\right) d t_{l} \cdots d t_{1}, \quad n \in \mathbb{N} .
\end{aligned}
$$


By (4.23), (4.19), and (4.29) we have from (4.30) that

$$
\left\|\Pi_{\alpha \alpha_{1}}^{(l)}\left(t, t_{1}, \ldots, t_{l} ; \mathbb{B}\right)\right\| \leq\left(\frac{l}{e T_{\delta}}\right)^{l},
$$

holding for all $l=1, \ldots, n$. This yields

$$
\begin{aligned}
\| & Q_{\alpha \alpha_{1}}^{(n)}(t ; \mathbb{B})-Q_{\alpha \alpha_{1}}^{(n-1)}(t ; \mathbb{B}) \| \\
& \leq \int_{0}^{t} \int_{0}^{t_{1}} \cdots \int_{0}^{t_{n-1}}\left\|\Pi_{\alpha \alpha_{1}}^{(n)}\left(t, t_{1}, \ldots, t_{l} ; \mathbb{B}\right)\right\| d t_{n} \cdots d t_{1} \\
& \leq \frac{1}{n !}\left(\frac{n}{e}\right)^{n}\left(\frac{T}{T_{\delta}}\right)^{n},
\end{aligned}
$$

hence,

$$
\forall t \in[0, T] \quad Q_{\alpha \alpha_{1}}^{(n)}(t ; \mathbb{B}) \rightarrow Q_{\alpha \alpha_{1}}(t ; \mathbb{B}) \in \mathcal{L}\left(\mathcal{K}_{\alpha_{1}}, \mathcal{K}_{\alpha}\right), \text { as } n \rightarrow+\infty .
$$

This proves claim $(i)$ of the lemma. The proof of claim (ii) follows by the fact that the mentioned above convergence is uniform on $[0, T]$. The estimate (4.26) readily follows from that in (4.34). Now by (4.30) and (4.32) we obtain

$$
\frac{d}{d t} Q_{\alpha_{2} \alpha_{1}}^{(n)}(t ; \mathbb{B})=\left(A_{b}^{\Delta}\right)_{\alpha_{2} \alpha} Q_{\alpha \alpha_{1}}^{(n)}(t ; \mathbb{B})+B_{\alpha_{2} \alpha} Q_{\alpha \alpha_{1}}^{(n-1)}(t ; \mathbb{B}), \quad n \in \mathbb{N} .
$$

Then the continuous differentiability of the limit and (4.27) follow by standard arguments.

Remark 4.7 By (4.30), (4.33), and Lemma 4.5 we have that

$$
\forall k \in \mathcal{K}_{\alpha_{1}}^{+} \quad Q_{\alpha_{2} \alpha_{1}}\left(t ; B_{2, b}^{\Delta}\right) k \in \mathcal{K}_{\alpha_{2}}^{+}, \quad t \in\left[0, T\left(\alpha_{2}, \alpha_{1} ; B_{2}^{\Delta}\right)\right) .
$$

At the same time, $Q_{\alpha_{2} \alpha_{1}}\left(t ; B_{b}^{\Delta}\right)$ is not positive, see (3.10) and Remark 4.4.

\subsection{The Proof of Theorem 3.3}

First we prove that the problem (3.17) has a unique solution on a bounded time interval.

Lemma 4.8 For each $\alpha_{2}>\alpha_{1}>-\log \vartheta$, the problem (3.17) with $k_{0} \in \mathcal{K}_{\alpha_{1}}$ has a unique solution $k_{t} \in \mathcal{K}_{\alpha_{2}}$ on the time interval $\left[0, T\left(\alpha_{2}, \alpha_{1}, B_{b}^{\Delta}\right)\right)$. The solution has the property: $k_{t}(\emptyset)=1$ for all $t \in\left[0, T\left(\alpha_{2}, \alpha_{1}, B_{b}^{\Delta}\right)\right)$.

Proof For each $t \in\left[0, T\left(\alpha_{2}, \alpha_{1}, B_{b}^{\Delta}\right)\right)$, one finds $\alpha \in\left(\alpha_{1}, \alpha_{2}\right)$ such that also $t \in$ $\left[0, T\left(\alpha, \alpha_{1}, B_{b}^{\Delta}\right)\right)$. Then by claim (i) of Lemma 4.5 and (3.15)

$$
k_{t}:=Q_{\alpha \alpha_{1}}\left(t ; B_{b}^{\Delta}\right) k_{0}
$$

lies in $\mathcal{D}_{\alpha_{2}}^{\Delta}$. By (4.27) the derivative of $k_{t} \in \mathcal{K}_{\alpha_{2}}$ is

$$
\frac{d}{d t} k_{t}=\left(\left(A_{b}^{\Delta}\right)_{\alpha_{2} \alpha}+\left(B_{b}^{\Delta}\right)_{\alpha_{2} \alpha}\right) k_{t}=L_{\alpha_{2} \alpha}^{\Delta} k_{t}
$$

Hence, $k_{t}$ is a solution of (3.17), see (3.16). Moreover, $k_{t}(\emptyset)=1$ since $k_{0}(\emptyset)=1$, see (2.12), and

$$
\left(\frac{d}{d t} k_{t}\right)(\emptyset)=\left(L_{\alpha}^{\Delta} k_{t}\right)(\emptyset)=0,
$$


see (3.8) - (3.10). To prove the stated uniqueness assume that $\tilde{k}_{t} \in \mathcal{D}_{\alpha_{2}}^{\Delta}$ is another solution of (3.17) with the same initial condition. Then for each $\alpha_{3}>\alpha_{2}, v_{t}:=k_{t}-\tilde{k}_{t}$ is a solution of (3.17) in $\mathcal{K}_{\alpha_{3}}$ with the zero initial condition. Here we assume that $t$ and $\alpha_{3}$ are such that $t<T\left(\alpha_{3}, \alpha_{1} ; B_{b}^{\Delta}\right)$. Clearly, $v_{t}$ also solves (3.17) in $\mathcal{K}_{\alpha_{2}}$. Thus, it can be written down in the following form

$$
v_{t}=\int_{0}^{t} \Sigma_{\alpha_{3} \alpha}(t-s)\left(B_{b}^{\Delta}\right)_{\alpha \alpha_{2}} v_{s} d s
$$

where $v_{t}$ on the left-hand side (resp. $v_{s}$ on the right-hand side) is considered as an element of $\mathcal{K}_{\alpha_{3}}$ (resp. $\left.\mathcal{K}_{\alpha_{2}}\right)$ and $\alpha \in\left(\alpha_{2}, \alpha_{3}\right)$. Indeed, one obtains (4.38) by integrating the equation, see (4.17),

$$
\frac{d}{d t} v_{t}=L_{\alpha_{3} \alpha_{2}}^{\Delta} v_{t}=\left(\left(A_{b}^{\Delta}\right)_{\alpha_{3} \alpha_{2}}+\left(B_{b}^{\Delta}\right)_{\alpha_{3} \alpha_{2}}\right) v_{t}, \quad v_{0}=0,
$$

in which the second summand is considered as a nonhomogeneous term, see (4.32). Let us show that for all $\left.t<T\left(\alpha_{2}, \alpha_{1} ; B_{b}^{\Delta}\right)\right), v_{t}=0$ as an element of $\mathcal{K}_{\alpha_{2}}$. In view of the embedding $\mathcal{K}_{\alpha_{2}} \hookrightarrow \mathcal{K}_{\alpha_{3}}$, cf. (2.17), this will follow from the fact that $v_{t}=0$ as an element of $\mathcal{K}_{\alpha_{3}}$. For a given $n \in \mathbb{N}$, we set $\epsilon=\left(\alpha_{3}-\alpha_{2}\right) / 2 n$ and $\alpha^{l}=\alpha_{2}+l \epsilon, l=0, \ldots, 2 n$. Then we repeatedly apply (4.38) and obtain

$$
\begin{aligned}
v_{t}= & \int_{0}^{t} \int_{0}^{t_{1}} \cdots \int_{0}^{t_{n-1}} \Sigma_{\alpha_{3} \alpha^{2 n-1}}\left(t-t_{1}\right)\left(B_{b}^{\Delta}\right)_{\alpha^{2 n-1} \alpha^{2 n-2}} \times \cdots \\
& \times \Sigma_{\alpha^{2} \alpha^{1}}\left(t_{n-1}-t_{n}\right)\left(B_{b}^{\Delta}\right)_{\alpha^{1} \alpha_{2}} v_{t_{n}} d t_{n} \cdots d t_{1} .
\end{aligned}
$$

Similarly as in (4.34) we then get from the latter, see (4.19), (4.20), and (4.23),

$$
\begin{aligned}
\left\|v_{t}\right\|_{\alpha_{3}} & \leq \frac{t^{n}}{n !} \prod_{l=1}^{n}\left\|\left(B_{b}^{\Delta}\right)_{\alpha^{2 l-1} \alpha^{2 l-2} \|} \sup _{s \in[0, t]}\right\| v_{s} \|_{\alpha_{2}} \\
& \leq \frac{1}{n !}\left(\frac{n}{e}\right)^{n}\left(\frac{2 t \beta\left(\alpha_{3} ; B_{b}^{\Delta}\right)}{\alpha_{3}-\alpha_{2}}\right)^{n} \sup _{s \in[0, t]}\left\|v_{s}\right\|_{\alpha_{2}} .
\end{aligned}
$$

This implies that $v_{t}=0$ for $t<\left(\alpha_{3}-\alpha_{2}\right) / 2 \beta\left(\alpha_{3} ; B_{b}^{\Delta}\right)$. To prove that $v_{t}=0$ for all $t$ of interest one has to repeat the above procedure appropriate number of times.

To make the next step we need the following result, the proof of which will be done in Sect. 5 below.

Lemma 4.9 (Identification Lemma) For each $\alpha_{2}>\alpha_{1}>-\log \vartheta$, there exists $\tau\left(\alpha_{2}, \alpha_{1}\right) \in$ $\left(0, T\left(\alpha_{2}, \alpha_{1} ; B_{b}^{\Delta}\right)\right)$ such that $Q_{\alpha_{2} \alpha_{1}}\left(t ; B_{b}^{\Delta}\right): \mathcal{K}_{\alpha_{1}}^{\star} \rightarrow \mathcal{K}_{\alpha_{2}}^{\star}$ for each $t \in\left[0, \tau\left(\alpha_{2}, \alpha_{1}\right)\right]$, see (2.18) and Lemma 4.5.

In the light of Proposition 2.3, Lemma 4.9 claims that for $t \in\left[0, \tau\left(\alpha_{2}, \alpha_{1}\right)\right]$, the solution $k_{t}$ as in Lemma 4.8 is the correlation function of a unique sub-Poissonian state $\mu_{t}$ whenever $k_{0}=k_{\mu_{0}}$ for some $\mu_{0} \in \mathcal{P}_{\mathrm{sP}}$.

To complete the proof of Theorem 4.2 we need the following result. Recall that $\mathcal{K}_{\alpha}^{\star} \subset \mathcal{K}_{\alpha}^{+}$, $\alpha \in \mathbb{R}$, see (2.19).

Lemma 4.10 Let $\alpha_{2}, \alpha_{1}$, and $\tau\left(\alpha_{2}, \alpha_{1}\right)$ be as in Lemma 4.9. Then there exists positive $\tau_{1}\left(\alpha_{2}, \alpha_{1}\right) \leq \tau\left(\alpha_{2}, \alpha_{1}\right)$ such that, for each $t \in\left[0, \tau_{1}\left(\alpha_{2}, \alpha_{1}\right)\right]$ and arbitrary $k_{0} \in \mathcal{K}_{\alpha_{1}}^{\star}$ the following holds, cf. (4.20) and Remark 4.4,

$$
0 \leq\left(Q_{\alpha_{2} \alpha_{1}}\left(t ; B_{b}^{\Delta}\right) k_{0}\right)(\eta) \leq\left(Q_{\alpha_{2} \alpha_{1}}\left(t ; B_{2, b}^{\Delta}\right) k_{0}\right)(\eta), \quad \eta \in \Gamma_{0} .
$$


Proof The left-hand inequality in (4.40) follows directly by Lemma 4.9. By Lemma $4.8 k_{t}$ as in (4.37) solves (3.17) in $\mathcal{K}_{\alpha_{2}}$. Set

$$
L_{2}^{\Delta}=A^{\Delta}+B_{2}^{\Delta}=A_{b}^{\Delta}+B_{2, b}^{\Delta},
$$

where $A^{\Delta}, B_{2}^{\Delta}$ and $A_{b}^{\Delta}, B_{2, b}^{\Delta}$ are as in (3.9), (3.10) and (4.15), (4.16), respectively. Then we introduce $\left(\left(L_{2}^{\Delta}\right)_{\alpha}, \mathcal{D}_{\alpha}^{\Delta}\right)$ and $\left(L_{2}^{\Delta}\right)_{\alpha \alpha^{\prime}}$ as in Sect. 3.2. By claims $(i)$ and (iv) of Lemma 4.5 we have that

$$
u_{t}:=Q_{\alpha \alpha_{1}}\left(t ; B_{2, b}^{\Delta}\right) k_{0}, \quad \alpha \in\left(\alpha_{1}, \alpha_{2}\right),
$$

solves the problem

$$
\frac{d}{d t} u_{t}=\left(L_{2}^{\Delta}\right)_{\alpha_{2}} u_{t}, \quad u_{0}=k_{0},
$$

on the time interval $\left[0, T\left(\alpha_{2}, \alpha_{1} ; B_{2, b}^{\Delta}\right)\right)$. Note that

$$
T\left(\alpha_{2}, \alpha_{1} ; B_{b}^{\Delta}\right) \leq T\left(\alpha_{2}, \alpha_{1} ; B_{2, b}^{\Delta}\right),
$$

see (4.20) and (4.24). Take $\alpha, \alpha^{\prime} \in\left(\alpha_{1}, \alpha_{2}\right), \alpha^{\prime}<\alpha$, and pick positive $\tau_{1} \leq \tau\left(\alpha_{2}, \alpha_{1}\right)$ such that

$$
\tau_{1}=\tau_{1}\left(\alpha_{2}, \alpha_{1}\right)<\min \left\{T\left(\alpha_{2}, \alpha ; B_{b}^{\Delta}\right) ; T\left(\alpha^{\prime}, \alpha_{1} ; B_{2, b}^{\Delta}\right)\right\} .
$$

By (4.42) the difference $u_{t}-k_{t} \in \mathcal{K}_{\alpha_{2}}$ can be written down in the form

$$
u_{t}-k_{t}=\int_{0}^{t} Q_{\alpha_{2} \alpha}\left(t-s ; B_{2, b}^{\Delta}\right)\left(-B_{1}^{\Delta}\right)_{\alpha \alpha^{\prime}} k_{s} d s,
$$

where $t \leq \tau_{1}$ and the operator $\left(-B_{1}^{\Delta}\right)_{\alpha \alpha^{\prime}}$ is positive with respect to the cone (2.19), see (3.10) and (3.13). In (4.43), $k_{s} \in \mathcal{K}_{\alpha^{\prime}}$ and $Q_{\alpha_{2} \alpha}\left(t-s ; B_{2, b}^{\Delta}\right) \in \mathcal{L}\left(\mathcal{K}_{\alpha}, \mathcal{K}_{\alpha_{2}}\right)$ for all $s \in\left[0, \tau_{1}\right]$. Since $Q_{\alpha_{2} \alpha}\left(t-s ; B_{2, b}^{\Delta}\right)$ is also positive, see Remark 4.4, and $k_{s} \in \mathcal{K}_{\alpha^{\prime}}^{\star} \subset \mathcal{K}_{\alpha^{\prime}}^{+}$(by (4.37) and Lemma 4.9), we have $u_{t}-k_{t} \in \mathcal{K}_{\alpha_{2}}^{+}$for $t \leq \tau_{1}\left(\alpha_{2}, \alpha_{1}\right)$, which yields (4.40).

Corollary 4.11 Let $\alpha_{2}, \alpha_{1}$, and $\tau_{1}\left(\alpha_{2}, \alpha_{1}\right)$ be as in Lemma 4.10. Then the following holds for all $t \leq \tau_{1}\left(\alpha_{2}, \alpha_{1}\right)$

$$
\left\|k_{t}\right\|_{\alpha_{2}}=\left\|Q_{\alpha_{2} \alpha_{1}}\left(t ; B_{b}^{\Delta}\right) k_{0}\right\|_{\alpha_{2}} \leq \frac{\left(\alpha_{2}-\alpha_{1}\right)\left\|k_{0}\right\|_{\alpha_{1}}}{\alpha_{2}-\alpha_{1}-t\left(\left\langle a^{+}\right\rangle+b\right)} .
$$

Proof Apply (4.40) and then (4.20) and (4.24).

Proof of Theorem 3.3 Let $\alpha_{0}>-\log \vartheta$ be such that $k_{\mu_{0}} \in \mathcal{K}_{\alpha_{0}}$, cf, (2.17). Then by Lemma 4.8 we have that for each $\alpha_{1}>\alpha_{0}$ and $\alpha \in\left(\alpha_{0}, \alpha_{1}\right)$,

$$
k_{t}:=Q_{\alpha \alpha_{0}}\left(t ; B_{b}^{\Delta}\right) k_{0} \in \mathcal{K}_{\alpha}^{\star}, \quad t \leq \tau_{1}\left(\alpha_{1}, \alpha_{0}\right),
$$

solves (3.17) in $\mathcal{K}_{\alpha_{1}}$. Its continuation to an arbitrary $t>0$ follows by (4.44) in a standard way.

\subsection{The Proof of Theorem 3.4}

\subsubsection{Case $\left\langle a^{+}\right\rangle>0$ and $m \in\left[0,\left\langle a^{+}\right\rangle\right]$}

The proof will be done by picking the corresponding bounds for $u_{t}$ defined in (4.41) with $k_{0}=k_{\mu_{0}} \in \mathcal{K}_{\alpha_{0}}^{\star}$. Recall that, for $\alpha_{1}>\alpha_{0}, u_{t} \in \mathcal{K}_{\alpha_{1}}$ for $t<T\left(\alpha_{1}, \alpha_{0} ; B_{2, b}^{\Delta}\right)$. For a given $\delta \leq m$, let us choose the value of $C_{\delta}$. The first condition is that 


$$
C_{\delta}^{|\eta|} \geq k_{0}(\eta)
$$

Next, if (3.5) holds with a given $\vartheta>0$ and $b=0$, we take any $\delta \leq m$ and $C_{\delta} \geq 1 / \vartheta$ such that also (4.45) holds. If (3.5) holds with $b>0$, we take any $\delta<m$ and then $C_{\delta} \geq b /(m-\delta) \vartheta$ such that also (4.45) holds. In all this cases, by Proposition 3.6 we have that

$$
E^{-}(\eta)-\frac{1}{C_{\delta}} E^{+}(\eta) \geq-(m-\delta)|\eta|, \quad \eta \in \Gamma_{0} .
$$

Let $r_{t}(\eta)$ denote the right-hand side of (3.18). For $\alpha_{1}>\alpha_{0}$, we take $\alpha, \alpha^{\prime} \in\left(\alpha_{0}, \alpha_{1}\right), \alpha^{\prime}<\alpha$ and then consider

$$
\begin{aligned}
v_{t} & :=Q_{\alpha_{1} \alpha_{0}}\left(t ; B_{2, b}^{\Delta}\right) r_{0} \\
& =r_{t}+\int_{0}^{t} Q_{\alpha_{1} \alpha}\left(t-s ; B_{2, b}^{\Delta}\right) D_{\alpha \alpha^{\prime}} r_{s} d s,
\end{aligned}
$$

where

$$
t \leq \tau_{2}:=\min \left\{\frac{\alpha^{\prime}-\alpha_{0}}{\left\langle a^{+}\right\rangle-\delta} ; T\left(\alpha_{1}, \alpha ; B_{2, b}^{\Delta}\right)\right\} .
$$

The operator $D$ in (4.47) is

$$
\begin{gathered}
\left(D_{\alpha \alpha^{\prime}} r_{s}\right)(\eta)=\left[-m|\eta|-E^{-}(\eta)+\frac{1}{C_{\delta}} \exp \left(-\left(\left\langle a^{+}\right\rangle-\delta\right) s\right) E^{+}(\eta)\right. \\
\quad+\delta|\eta|] r_{s}(\eta) \leq 0, \quad \eta \in \Gamma_{0} .
\end{gathered}
$$

The latter inequality holds for all $s \in\left[0, \tau_{2}\right]$, see (4.46), and all $m \in\left[0,\left\langle a^{+}\right\rangle\right]$and $\delta<m$. Then by (4.36) we obtain from (4.41), the first line of (4.47), and (4.45) that

$$
u_{t}(\eta) \leq v_{t}(\eta), \quad t<T\left(\alpha_{1}, \alpha_{0} ; B_{2, b}^{\Delta}\right) .
$$

Then by the second line of (4.47) and (4.49) we get that for $t \leq \tau_{2}$, see (4.48), the following holds

$$
u_{t}(\eta) \leq v_{t}(\eta) \leq r_{t}(\eta), \quad \eta \in \Gamma_{0} .
$$

The continuation of the latter inequality to bigger values of $t$ is straightforward. This completes the proof for this case.

\subsubsection{Case $\left\langle a^{+}\right\rangle>0$ and $m>\left\langle a^{+}\right\rangle$}

Take $\varepsilon \in\left(0, m-\left\langle a^{+}\right\rangle\right)$and then set

$$
\vartheta_{\varepsilon}=\vartheta\left(1-\frac{\varepsilon+2\left\langle a^{+}\right\rangle}{2 m}\right) .
$$

Thereafter, choose $C_{\varepsilon} \geq 1 / \vartheta_{\varepsilon}$ such that

$$
C_{\varepsilon}^{|\eta|} \geq k_{0}(\eta), \quad \eta \in \Gamma_{0} .
$$

Then, cf. (4.46),

$$
E^{-}(\eta)-\frac{1}{C_{\varepsilon}} E^{+}(\eta) \geq-\left(m-\left\langle a^{+}\right\rangle-\varepsilon / 2\right)|\eta|, \quad \eta \in \Gamma_{0} .
$$


Let now $r_{t}$ stand for the right-hand side of (3.19). Then the second line of (4.47) holds with $D_{\alpha \alpha^{\prime}}$ replaced by $D_{\alpha \alpha^{\prime}}^{\varepsilon}$. By definition the latter is such that: (a) $\left(D_{\alpha \alpha^{\prime}}^{\varepsilon} r_{s}\right)(\emptyset)=0$;

$$
\text { (b) }\left(D_{\alpha \alpha^{\prime}}^{\varepsilon} r_{s}\right)(\{x\})=-\left(m-\left\langle a^{+}\right\rangle-\varepsilon\right) r_{s}(\{x\}) \leq 0 \text {, }
$$

and, for $|\eta| \geq 2$, see (4.50),

$$
\text { (c) } \begin{aligned}
\left(D_{\alpha \alpha^{\prime}}^{\varepsilon} r_{s}\right)(\eta) & =\left[\varepsilon-m|\eta|-E^{-}(\eta)+\frac{1}{C_{\varepsilon}} E^{+}(\eta)+\left\langle a^{+}\right\rangle|\eta|\right] r_{s}(\eta) \\
& \leq \varepsilon(1-|\eta| / 2) r_{s}(\eta) \leq 0 .
\end{aligned}
$$

This yields (3.19) and thus completes the proof for this case.

\subsubsection{The Remaining Cases}

For $\left\langle a^{+}\right\rangle=0$ and $t>0$, we set

$$
\left(Q_{\alpha \alpha^{\prime}}^{(0)}(t) u\right)(\eta)=\exp [-t E(\eta)] u(\eta),
$$

where $\alpha^{\prime}<\alpha$ and $u \in \mathcal{K}_{\alpha^{\prime}}$. Then, cf. Lemma 4.5, $Q_{\alpha \alpha^{\prime}}^{(0)}(t): \mathcal{K}_{\alpha^{\prime}} \rightarrow \mathcal{K}_{\alpha}$ continuously, and the map

$$
[0,+\infty) \ni t \mapsto Q_{\alpha \alpha^{\prime}}^{(0)}(t) \in \mathcal{L}\left(\mathcal{K}_{\alpha^{\prime}}, \mathcal{K}_{\alpha}\right)
$$

is continuous and such that, cf. (4.27),

$$
\frac{d}{d t} Q_{\alpha^{\prime \prime} \alpha^{\prime}}^{(0)}(t)=\left(A_{1}^{\Delta}\right)_{\alpha^{\prime \prime} \alpha} Q_{\alpha \alpha^{\prime}}^{(0)}(t), \quad \alpha^{\prime \prime}>\alpha
$$

where $\left(A_{1}^{\Delta}\right)_{\alpha^{\prime \prime} \alpha}$ is defined in (3.9) and (3.11). Now we set $u_{t}=Q_{\alpha \alpha_{0}}^{(0)}(t) k_{\mu_{0}}$ and obtain from (4.51) and (4.52), similarly as in (4.43),

$$
u_{t}-k_{t}=\int_{0}^{t} Q_{\alpha \alpha_{1}}^{(0)}(t)\left(-B_{1}^{\Delta}\right)_{\alpha_{1} \alpha_{2}} k_{s} d s \geq 0,
$$

which yields (3.20).

To prove that $r_{t}(\eta):=\vartheta^{-|\eta|}, t \geq 0$, is a stationary solution we set

$$
k_{t}=Q_{\alpha \alpha_{0}}\left(t ; B_{b}^{\Delta}\right) r_{0},
$$

where $\alpha_{0}>-\log \vartheta$ and $\alpha>\alpha_{0}$. Then the following holds, cf. (4.43),

$$
k_{t}=r_{t}+\int_{0}^{t} Q_{\alpha \alpha_{2}}\left(t-s ; B_{b}^{\Delta}\right) L_{\alpha_{2} \alpha_{1}}^{\Delta} r_{s} d s,
$$

where $\alpha_{1}<\alpha_{2}$ are taken from $\left(\alpha_{0}, \alpha\right)$. For the case considered, we have

$$
L_{\alpha_{2} \alpha_{1}}^{\Delta} r_{s}=L_{\alpha_{2} \alpha_{1}}^{\Delta} r_{0}=0
$$

which completes the proof for this case. 


\section{The Proof of the Identification Lemma}

To prove Lemma 4.9 we use Proposition 2.3. Note that the solution mentioned in Lemma 4.8 already has properties (ii) and (iii) of (2.12), cf. (2.14). Thus, it remains to prove that also (i) holds. We do this as follows. First, we approximate the evolution $k_{0} \mapsto k_{t}$ established in Lemma 4.8 by evolutions $k_{0 \text {,app }} \mapsto k_{t \text {,app }}$ such that $k_{t \text {, app }}$ has property (i). Then we prove that for each $G \in B_{\mathrm{bs}}^{\star}\left(\Gamma_{0}\right),\left\langle\left\langle G, k_{t}\right.\right.$,app $\left.\rangle\right\rangle \rightarrow\left\langle\left\langle G, k_{t}\right\rangle\right\rangle$ as the approximations are eliminated. The limiting transition is based on the representation $\left\langle\left\langle G, k_{t, \text { app }}\right\rangle\right\rangle=\left\langle\left\langle G_{t}, k_{0 \text {,app }}\right\rangle\right\rangle$ in which we use the so called predual evolution $G \mapsto G_{t}$. Then we just show that $\left\langle\left\langle G_{t}, k_{0 \text {,app }}\right\rangle\right\rangle \rightarrow\left\langle\left\langle G_{t}, k_{0}\right\rangle\right\rangle$.

\subsection{The Predual Evolution}

The aim of this subsection is to construct the evolution $B_{\mathrm{loc}}\left(\Gamma_{0}\right) \ni G_{0} \mapsto G_{t} \in \mathcal{G}_{\alpha_{1}}$, see (4.1) and (4.2), such that, for each $\alpha>\alpha_{1}$ and $k_{0} \in \mathcal{K}_{\alpha_{1}}$, the following holds, cf. (4.37),

$$
\left\langle G_{0}, Q_{\alpha \alpha_{1}}\left(t ; B_{b}^{\Delta}\right) k_{0}\right\rangle=\left\langle\left\langle G_{t}, k_{0}\right\rangle\right\rangle
$$

where $b \geq 0$ and $B_{b}^{\Delta}$ are as in (3.5) and (4.16), respectively. Let us define the action of $B_{b}$ on appropriate $G: \Gamma_{0} \rightarrow \mathbb{R}$ via the duality

$$
\left\langle\left\langle G, B_{b}^{\Delta} k\right\rangle\right\rangle=\left\langle\left\langle B_{b} G, k\right\rangle\right\rangle .
$$

Similarly as in (4.16) we then get

$$
\begin{aligned}
\left(B_{b} G\right)(\eta)=b|\eta| G(\eta) & +\int_{\mathbb{R}^{d}} \sum_{x \in \eta} a^{+}(x-y) G(\eta \backslash x \cup y) d y \\
& -\sum_{x \in \eta} E^{-}(x, \eta \backslash x) G(\eta \backslash x) .
\end{aligned}
$$

For $\alpha_{2}>\alpha_{1}$, let $\left(B_{b}\right)_{\alpha_{1} \alpha_{2}}$ be the bounded linear operator from $\mathcal{G}_{\alpha_{2}}$ to $\mathcal{G}_{\alpha_{1}}$ the action of which is defined in (5.2). As in estimating the norm of $B_{b}^{\Delta}$ in (4.18) one then gets

$$
\left\|\left(B_{b}\right)_{\alpha_{1} \alpha_{2}}\right\| \leq \frac{\left\langle a^{+}\right\rangle+b+\left\langle a^{-}\right\rangle e^{\alpha_{2}}}{e\left(\alpha_{2}-\alpha_{1}\right)} .
$$

For the same $\alpha_{2}$ and $\alpha_{1}$, let $S_{\alpha_{1} \alpha_{2}}(t)$ be the restriction to $\mathcal{G}_{\alpha_{2}}$ of the corresponding element of the semigroup mentioned in Lemma 4.2. Then $S_{\alpha_{1} \alpha_{2}}(t)$ acts as a bounded contraction from $\mathcal{G}_{\alpha_{2}}$ to $\mathcal{G}_{\alpha_{1}}$.

Now for a given $l \in \mathbb{N}$ and $\alpha, \alpha_{1}$ as in (5.1), let $\delta$ and $\alpha^{s}, s=0, \ldots, 2 l+1$, be as in (4.29). Then for $t>0$ and $\left(t, t_{1}, \ldots, t_{l}\right) \in \mathcal{T}_{l}$, see (4.28), we define, cf. (4.30),

$$
\begin{aligned}
\Omega_{\alpha_{1} \alpha}^{(l)}\left(t, t_{1}, \ldots, t_{l}\right)= & S_{\alpha_{1} \alpha^{1}}\left(t_{l}\right)\left(B_{b}\right)_{\alpha^{1} \alpha^{2}} S_{\alpha^{2} \alpha^{3}}\left(t_{l-1}-t_{l}\right) \times \cdots \\
& \times\left(B_{b}\right)_{\alpha^{2 s-1} \alpha^{2 s}} S_{\alpha^{2 s} \alpha^{2 s+1}}\left(t_{l-s}-t_{l-s+1}\right) \cdots\left(B_{b}\right)_{\alpha^{2 l-1} \alpha^{2 l}} S_{\alpha^{2 l} \alpha}\left(t-t_{1}\right) .
\end{aligned}
$$

As in Proposition 4.6, one shows that the map

$$
\mathcal{T}_{l} \ni\left(t, t_{1}, \ldots, t_{l}\right) \mapsto \Omega_{\alpha_{1} \alpha}^{(l)}\left(t, t_{1}, \ldots, t_{l}\right) \in \mathcal{L}\left(\mathcal{G}_{\alpha}, \mathcal{G}_{\alpha_{1}}\right)
$$

is continuous. Define

$$
H_{\alpha_{1} \alpha}^{(n)}(t)=S_{\alpha_{1} \alpha}(t)+\sum_{l=1}^{n} \int_{0}^{t} \int_{0}^{t_{1}} \ldots \int_{0}^{t_{l-1}} \Omega_{\alpha_{1} \alpha}^{(l)}\left(t, t_{1}, \ldots, t_{l}\right) d t_{l} \cdots d t_{1} .
$$


Lemma 5.1 For each $T \in\left(0, T\left(\alpha, \alpha_{1} ; B_{b}^{\Delta}\right)\right)$, see (4.24) and (4.20), the sequence of operators defined in (5.4) converges in $\mathcal{L}\left(\mathcal{G}_{\alpha}, \mathcal{G}_{\alpha_{1}}\right)$ to a certain $H_{\alpha_{1} \alpha}(t)$ uniformly on $[0, T]$, and for each $G_{0} \in \mathcal{G}_{\alpha}$ and $k_{0} \in \mathcal{K}_{\alpha_{1}}$ the following holds

$$
\left.\| G_{0}, Q_{\alpha \alpha_{1}}\left(t ; B_{b}^{\Delta}\right) k_{0}\right\rangle=\left\langle\left\langle H_{\alpha_{1} \alpha}(t) G_{0}, k_{0}\right\rangle\right\rangle, \quad t \in[0, T] .
$$

Proof For the operators defined in (5.4), similarly as in (4.35) we get the following estimate

$$
\left\|H_{\alpha_{1} \alpha}^{(n)}(t)-H_{\alpha_{1} \alpha}^{(n-1)}(t)\right\| \leq \frac{1}{n !}\left(\frac{n}{e}\right)^{n}\left(\frac{T}{T_{\delta}}\right)^{n},
$$

which yields the convergence stated in the lemma. By direct inspection one gets that

$$
\| G_{0}, Q_{\alpha \alpha_{1}}^{(n)}\left(t ; B^{\Delta}\right) k_{0} \rrbracket=\left\langle H_{\alpha_{1} \alpha}^{(n)}(t) G_{0}, k_{0} \|,\right.
$$

see (4.33). Then (5.5) is obtained from the latter in the limit $n \rightarrow+\infty$. Similarly as in (4.26), for the limiting operator the following estimate holds

$$
\left\|H_{\alpha_{1} \alpha}(t)\right\| \leq \frac{T\left(\alpha, \alpha_{1} ; B_{b}^{\Delta}\right)}{T\left(\alpha, \alpha_{1} ; B_{b}^{\Delta}\right)-t} .
$$

\subsection{An Auxiliary Model}

The approximations mentioned at the beginning of this section employ also an auxiliary model, which we introduce and study now. For this model, we construct three kinds of evolutions. The first one is $k_{0} \mapsto k_{t} \in \mathcal{K}_{\alpha}$ obtained as in Lemma 4.8. Another evolution $q_{0} \mapsto$ $q_{t} \in \mathcal{G}_{\omega}$ is constructed in such a way that $q_{t}$ is positive definite in the sense that $\left\langle\left\langle G, q_{t}\right\rangle\right\rangle \geq 0$ for all $G \in B_{\mathrm{bs}}^{\star}\left(\Gamma_{0}\right)$. These evolutions, however, take place in different spaces. To relate them to each other we construct one more evolution, $u_{0} \mapsto u_{t}$, which takes place in the intersection of the mentioned Banach spaces. The aim is to show that $k_{t}=u_{t}=q_{t}$ and thereby to get the desired property of $k_{t}$. Thereafter, we prove the convergence mentioned above.

\subsubsection{The Model}

The function

$$
\varphi_{\sigma}(x)=\exp \left(-\sigma|x|^{2}\right), \quad \sigma>0, \quad x \in \mathbb{R}^{d},
$$

has the following evident properties

$$
\bar{\varphi}_{\sigma}:=\int_{\mathbb{R}^{d}} \varphi(x) d x<\infty, \quad \varphi_{\sigma}(x) \leq 1, \quad x \in \mathbb{R}^{d} .
$$

The model we need is characterized by $L$ as in (1.4) with $E^{+}(x, \eta)$, cf. (1.5), replaced by

$$
E_{\sigma}^{+}(x, \eta)=\varphi_{\sigma}(x) E_{\sigma}^{+}(x, \eta)=\varphi_{\sigma}(x) \sum_{y \in \eta} a^{+}(x-y) .
$$

\subsubsection{The Evolution in $\mathcal{K}_{\alpha}$}

For the new model (with $E_{\sigma}^{+}$as in (5.9)), the operator $L^{\Delta, \sigma}$ corresponding to $L^{\Delta}$ takes the form, cf. (3.8) - (3.10) and (4.15) - (4.17),

$$
L^{\Delta, \sigma}=A^{\Delta, \sigma}+B^{\Delta, \sigma}=A_{b}^{\Delta, \sigma}+B_{b}^{\Delta, \sigma} .
$$


Here

$$
\begin{array}{ll}
A^{\Delta, \sigma}=A_{1}^{\Delta}+A_{2}^{\Delta, \sigma}, & A_{b}^{\Delta, \sigma}=A_{1, b}^{\Delta}+A_{2}^{\Delta, \sigma}, \\
B^{\Delta, \sigma}=B_{1}^{\Delta}+B_{2}^{\Delta, \sigma}, & B_{b}^{\Delta, \sigma}=B_{1}^{\Delta}+B_{2, b}^{\Delta, \sigma},
\end{array}
$$

where $A_{1}^{\Delta}, B_{1}^{\Delta}$, and $A_{1, b}^{\Delta}$ are the same as in (3.9), (3.10), and (4.15), respectively, and

$$
\begin{aligned}
& \left(A_{2}^{\Delta, \sigma} k\right)(\eta)=\sum_{x \in \eta} \varphi_{\sigma}(x) E^{+}(x, \eta \backslash x) k(\eta \backslash x), \\
& \left(B_{2}^{\Delta, \sigma} k\right)(\eta)=b|\eta| k(\eta)+\int_{\mathbb{R}^{d}} \sum_{x \in \eta} \varphi_{\sigma}(x) a^{+}(x-y) k(\eta \backslash x \cup y) d y .
\end{aligned}
$$

Note that these $A_{b}^{\Delta, \sigma}$ and $B_{b}^{\Delta, \sigma}$ define the corresponding bounded operators acting from $\mathcal{K}_{\alpha^{\prime}}$ to $\mathcal{K}_{\alpha}$ for each real $\alpha>\alpha^{\prime}$. As in (3.15) we then set

$$
\mathcal{D}_{\alpha}^{\Delta, \sigma}=\left\{k \in \mathcal{K}_{\alpha}: L^{\Delta, \sigma} k \in \mathcal{K}_{\alpha}\right\},
$$

and thus define the corresponding operator $\left(L_{\alpha}^{\Delta, \sigma}, \mathcal{D}_{\alpha}^{\Delta, \sigma}\right)$. Along with (3.17) we also consider

$$
\frac{d}{d t} k_{t}=L_{\alpha}^{\Delta, \sigma} k_{t},\left.\quad k_{t}\right|_{t=0}=k_{0} \in \mathcal{D}_{\alpha}^{\Delta, \sigma}
$$

By the literal repetition of the construction used in the proof of Lemma 4.5 one obtains the operators $Q_{\alpha \alpha^{\prime}}^{\sigma}\left(t ; B_{b}^{\Delta, \sigma}\right),\left(\alpha, \alpha^{\prime}, t\right) \in \mathcal{A}\left(B_{b}^{\Delta}\right)$, see (4.25), the norm of which satisfies, cf. (4.26),

$$
\left\|Q_{\alpha \alpha^{\prime}}^{\sigma}\left(t ; B_{b}^{\Delta, \sigma}\right)\right\| \leq \frac{T\left(\alpha, \alpha^{\prime} ; B_{b}^{\Delta}\right)}{T\left(\alpha, \alpha^{\prime} ; B_{b}^{\Delta}\right)-t},
$$

which is uniform in $\sigma$.

Lemma 5.2 Let $\alpha_{1}$ and $\alpha_{2}$ be as in Lemma 4.8. Then for a given $k_{0} \in \mathcal{K}_{\alpha_{1}}$, the unique solution of (5.14) in $\mathcal{K}_{\alpha_{2}}$ is given by

$$
k_{t}=Q_{\alpha \alpha_{1}}^{\sigma}\left(t ; B_{b}^{\Delta, \sigma}\right) k_{0}, \quad \alpha \in\left(\alpha_{1}, \alpha_{2}\right), \quad t<T\left(\alpha_{2}, \alpha_{1} ; B_{b}^{\Delta}\right) .
$$

Proof Repeat the proof of Lemma 4.8.

\subsubsection{The Evolution in $\mathcal{U}_{\sigma, \alpha}$}

For $\varphi_{\sigma}$ as in (5.7) we set

$$
e\left(\varphi_{\sigma} ; \eta\right)=\prod_{x \in \eta} \varphi_{\sigma}(x), \quad \eta \in \Gamma_{0},
$$

and introduce the following Banach space. For $u: \Gamma_{0} \rightarrow \mathbb{R}$, we define the norm, cf. (2.14),

$$
\|u\|_{\sigma, \alpha}=\underset{\eta \in \Gamma_{0}}{\operatorname{ess} \sup } \frac{|u(\eta)| \exp (-\alpha|\eta|)}{e\left(\varphi_{\sigma} ; \eta\right)} .
$$

Thereafter, set

$$
\mathcal{U}_{\sigma, \alpha}=\left\{u: \Gamma_{0} \rightarrow \mathbb{R}:\|u\|_{\sigma, \alpha}<\infty\right\} .
$$

By (5.7) and (2.14) we have that

$$
\|u\|_{\alpha} \leq\|u\|_{\sigma, \alpha}, \quad u \in \mathcal{U}_{\sigma, \alpha},
$$


which yields $\mathcal{U}_{\sigma, \alpha} \hookrightarrow \mathcal{K}_{\alpha}$. Moreover, as in (2.17) we also have that $\mathcal{U}_{\sigma, \alpha^{\prime}} \hookrightarrow \mathcal{U}_{\sigma, \alpha}$ for each real $\alpha>\alpha^{\prime}$.

Now let us define the operator $L_{\alpha, u}^{\Delta, \sigma}$ in $\mathcal{U}_{\sigma, \alpha}$ the action of which is described in (5.10) (5.12) and the domain is, cf. (5.13),

$$
\mathcal{D}_{\alpha, u}^{\Delta, \sigma}=\left\{u \in \mathcal{U}_{\sigma, \alpha}: L^{\Delta, \sigma} u \in \mathcal{U}_{\sigma, \alpha}\right\}
$$

Then we consider

$$
\frac{d}{d t} u_{t}=L_{\alpha, u}^{\Delta, \sigma} u_{t},\left.\quad u_{t}\right|_{t=0}=u_{0} \in \mathcal{D}_{\alpha, u}^{\Delta, \sigma} .
$$

Note that $\mathcal{U}_{\sigma, \alpha^{\prime \prime}} \subset \mathcal{D}\left(L_{\alpha, u}^{\Delta, \sigma}\right)$ for each $\alpha^{\prime \prime}<\alpha$, and

$$
\left(L_{\alpha, u}^{\Delta, \sigma}, \mathcal{D}_{\alpha, u}^{\Delta, \sigma}\right) \subset\left(L_{\alpha}^{\Delta, \sigma}, \mathcal{D}_{\alpha}^{\Delta, \sigma}\right) .
$$

Our aim now is to prove that the problem (5.19) with $u_{0} \in \mathcal{U}_{\sigma, \alpha_{1}}$ has a unique solution in $\mathcal{U}_{\sigma, \alpha_{2}}$, where $\alpha_{1}<\alpha_{2}$ are as in Lemma 4.8. To this end we first construct the semigroup analogous to that obtained in Lemma 4.2. Thus, in the predual space $\mathcal{G}_{\sigma, \alpha}$ equipped with the norm, cf. (4.2),

$$
|G|_{\sigma, \alpha}:=\int_{\Gamma_{0}}|G(\eta)| \exp (\alpha|\eta|) e\left(\varphi_{\sigma} ; \eta\right) \lambda(d \eta)
$$

we define the action of $A_{b}^{\sigma}$ as follows, cf. (4.5),

$$
\begin{aligned}
A_{b}^{\sigma} & =A_{1, b}+A_{2}^{\sigma} \\
\left(A_{2}^{\sigma} G\right)(\eta) & =\int_{\mathbb{R}^{d}} \varphi_{\sigma}(y) E^{+}(y, \eta) G(\eta \cup y) d y,
\end{aligned}
$$

and $A_{1, b}$ acts as in (4.5). Then we have, cf. (4.7),

$$
\begin{aligned}
& \left|A_{2}^{\sigma} G\right|_{\sigma, \alpha} \\
& \quad \leq \int_{\Gamma_{0}}\left(\int_{\mathbb{R}^{d}} \varphi_{\sigma}(y) E^{+}(y, \eta)|G(\eta \cup y)| d y\right) \exp (\alpha|\eta|) e\left(\varphi_{\sigma} ; \eta\right) \lambda(d \eta) \\
& \quad=\int_{\Gamma_{0}} e^{-\alpha}\left(\sum_{x \in \eta} E^{+}(x, \eta \backslash x)\right)|G(\eta)| \exp (\alpha|\eta|) e\left(\varphi_{\sigma} ; \eta\right) \lambda(d \eta) \\
& \quad \leq\left(e^{-\alpha} / \vartheta\right)\left|A_{1, b} G\right|_{\sigma, \alpha} .
\end{aligned}
$$

Now the existence of the substochastic semigroup $\left\{S_{\sigma, \alpha}(t)\right\}_{t \geq 0}$ generated by $\left(A_{b}^{\sigma}, \mathcal{D}_{\sigma, \alpha}\right)$ follows as in Lemma 4.2. Here, cf. (4.6),

$$
\mathcal{D}_{\sigma, \alpha}:=\left\{G \in \mathcal{G}_{\sigma, \alpha}: E_{b}(\cdot) G \in \mathcal{G}_{\sigma, \alpha}\right\} .
$$

Let $S_{\sigma, \alpha}^{\odot}(t)$ be the sun-dual to $S_{\sigma, \alpha}(t)$, cf. (4.11). Then for each $\alpha^{\prime}<\alpha$ and any $u \in \mathcal{U}_{\sigma, \alpha^{\prime}}$, the map

$$
[0,+\infty) \ni t \mapsto S_{\sigma, \alpha}^{\odot}(t) u \in \mathcal{U}_{\sigma, \alpha}
$$

is continuous, see Proposition 4.3. For real $\alpha^{\prime}<\alpha$ and $t>0$, let $\Sigma_{\alpha \alpha^{\prime}}^{\sigma, u}(t)$ be the restriction of $S_{\sigma, \alpha}^{\odot}(t)$ to $\mathcal{U}_{\sigma, \alpha^{\prime}}$. Then the map

$$
[0,+\infty) \ni t \mapsto \Sigma_{\alpha \alpha^{\prime}}^{\sigma, u}(t) \in \mathcal{L}\left(\mathcal{U}_{\sigma, \alpha^{\prime}}, \mathcal{U}_{\sigma, \alpha}\right)
$$

is continuous and such that, cf. (4.23),

$$
\left\|\Sigma_{\alpha \alpha^{\prime}}^{\sigma, u}(t)\right\| \leq 1, \quad t \geq 0 .
$$


Now we define $\left(B_{b}^{\Delta, \sigma}\right)_{\alpha \alpha^{\prime}}$ which acts from $\mathcal{U}_{\sigma, \alpha^{\prime}}$ to $\mathcal{U}_{\sigma, \alpha}$ according to (5.11) and (5.12). Then its norm satisfies

$$
\left\|\left(B_{b}^{\Delta, \sigma}\right)_{\alpha \alpha^{\prime}}\right\| \leq \frac{\left\langle a^{+}\right\rangle+b+\left\langle a^{-}\right\rangle e^{\alpha}}{e\left(\alpha-\alpha^{\prime}\right)} .
$$

In proving this we take into account that $\varphi_{\sigma}(x) \leq 1$ and repeat the arguments used in obtaining (4.18).

For real $\alpha_{2}>\alpha_{1}>-\log \vartheta$, we take $\alpha \in\left(\alpha_{1}, \alpha_{2}\right]$ and then pick $\delta<\alpha-\alpha_{1}$ as in the proof of Lemma 4.5. Next, for $l \in \mathbb{N}$ we divide $\left[\alpha_{1}, \alpha\right]$ into subintervals according to (4.29) and take $\left(t, t_{1}, \ldots, t_{l}\right) \in \mathcal{T}_{l}$, see (4.28). Then define, cf. (4.30),

$$
\begin{aligned}
\Pi_{\alpha \alpha_{1}}^{l, \sigma}\left(t, t_{1}, \ldots, t_{l}\right)= & \Sigma_{\alpha \alpha^{2 l}}^{\sigma, u}\left(t-t_{1}\right)\left(B_{b}^{\Delta, \sigma}\right)_{\alpha^{2 l} \alpha^{2 l-1}} \\
& \times \Sigma_{\alpha^{2 l-1} \alpha^{2 l-2}}^{\sigma, u}\left(t_{1}-t_{2}\right) \times \cdots \times \Sigma_{\alpha^{3} \alpha^{2}}^{\sigma, u}\left(t_{l-1}-t_{l}\right)\left(B_{b}^{\Delta, \sigma}\right)_{\alpha^{2} \alpha^{1}} \Sigma_{\alpha^{1} \alpha_{1}}^{\sigma, u}\left(t_{l}\right) .
\end{aligned}
$$

Thereafter, for $n \in \mathbb{N}$ we set, cf. (4.33),

$$
\begin{aligned}
U_{\alpha \alpha_{1}}^{(n)}(t)= & \Sigma_{\alpha \alpha_{1}}^{\sigma, u}(t) \\
& +\sum_{l=1}^{n} \int_{0}^{t} \int_{0}^{t_{1}} \cdots \int_{0}^{t_{n-1}} \Pi_{\alpha \alpha_{1}}^{l, \sigma}\left(t, t_{1}, \ldots, t_{l}\right) d t_{l} \cdots d t_{1} .
\end{aligned}
$$

By means of (5.21) and (5.22) we then prove that the sequence $\left\{U_{\alpha \alpha_{1}}^{(n)}(t)\right\}_{n \in \mathbb{N}}$ converges in $\mathcal{L}\left(\mathcal{U}_{\sigma, \alpha_{1}}, \mathcal{U}_{\sigma, \alpha}\right)$, uniformly on [0,T], $T<T\left(\alpha, \alpha_{1} ; B_{b}^{\Delta}\right)$, see (4.24) and (4.20). The limit $U_{\alpha \alpha_{1}}(t) \in \mathcal{L}\left(\mathcal{U}_{\sigma, \alpha_{1}}, \mathcal{U}_{\sigma, \alpha}\right)$ has the property, cf. (4.27),

$$
\frac{d}{d t} U_{\alpha_{2} \alpha_{1}}(t)=\left(\left(A_{b}^{\Delta, \sigma}\right)_{\alpha_{2} \alpha}+\left(B_{b}^{\Delta, \sigma}\right)_{\alpha_{2} \alpha}\right) U_{\alpha \alpha_{1}}(t)
$$

where $\left(A_{b}^{\Delta, \sigma}\right)_{\alpha_{2} \alpha} \in \mathcal{L}\left(\mathcal{U}_{\sigma, \alpha}, \mathcal{U}_{\sigma, \alpha_{2}}\right)$ is defined in (5.11) and (5.12), analogously to (5.22). Note that

$$
\forall u \in \mathcal{U}_{\sigma, \alpha} \quad L_{\alpha_{2}, u}^{\Delta, \sigma} u=\left(\left(A_{b}^{\Delta, \sigma}\right)_{\alpha_{2} \alpha}+\left(B_{b}^{\Delta, \sigma}\right)_{\alpha_{2} \alpha}\right) u,
$$

see (5.18). Now we can state the following analog of Lemma 4.8.

Lemma 5.3 Let $\alpha_{2}>\alpha_{1}>-\log \vartheta$ be as in Lemma 4.8. Then the problem (5.19) with $u_{0} \in \mathcal{U}_{\sigma, \alpha_{1}}$ has a unique solution $u_{t} \in \mathcal{U}_{\sigma, \alpha_{2}}$ on the time interval $\left[0, T\left(\alpha_{2}, \alpha_{1} ; B_{b}^{\Delta}\right)\right)$.

Proof Fix $T<T\left(\alpha_{2}, \alpha_{1} ; B_{b}^{\Delta}\right)$ and find $\alpha \in\left(\alpha_{1}, \alpha_{2}\right)$ such that also $T<T\left(\alpha^{\prime}, \alpha_{1} ; B_{b}^{\Delta}\right)$. Then, cf. (4.37),

$$
u_{t}:=U_{\alpha \alpha_{1}}(t) u_{0}
$$

is the solution in question, which can be checked by means of (5.23). Its uniqueness can be proved by the literal repetition of the corresponding arguments used in the proof of Lemma 4.8 .

Corollary 5.4 Let $k_{t}$ be the solution of the problem (5.14) with $k_{0} \in \mathcal{U}_{\sigma, \alpha_{1}}$ mentioned in Lemma 5.2. Then $k_{t}$ coincides with the solution mentioned in Lemma 5.3.

Proof Since $\left(L_{\alpha}^{\Delta, \sigma}, \mathcal{D}_{\alpha}^{\Delta, \sigma}\right)$ is an extension of $\left(L_{\alpha, u}^{\Delta, \sigma}, \mathcal{D}_{\alpha, u}^{\Delta, \sigma}\right)$, see (5.20), and the embedding $\mathcal{U}_{\sigma, \alpha} \hookrightarrow \mathcal{K}_{\alpha}$ is continuous, the solution as in (5.24) with $u_{0}=k_{0}$ satisfies also (5.14), and hence coincides with $k_{t}$ in view of the uniqueness stated in Lemma 5.2. 


\subsubsection{The Evolution in $\mathcal{G}_{\omega}$}

We recall that the space $\mathcal{G}_{\alpha}$ was introduced in (4.1), (4.2), where we used it as a predual space to $\mathcal{K}_{\alpha}$. Now we employ $\mathcal{G}_{\alpha}$ to get the positive definiteness mentioned at the beginning of this subsection. Here, however, we write $\mathcal{G}_{\omega}$ to show that we use it not as a predual space.

Let $L^{\Delta, \sigma}$ be as in (5.10). For $\omega \in \mathbb{R}$, we set, cf. (5.13) and (5.18),

$$
\mathcal{D}_{\omega}^{\Delta, \sigma}=\left\{q \in \mathcal{G}_{\omega}: L^{\Delta, \sigma} q \in \mathcal{G}_{\omega}\right\} .
$$

Then we define the corresponding operator $\left(L_{\omega}^{\Delta, \sigma}, \mathcal{D}_{\omega}^{\Delta, \sigma}\right)$ and consider the following Cauchy problem

$$
\frac{d}{d t} q_{t}=L_{\omega}^{\Delta, \sigma} q_{t},\left.\quad q_{t}\right|_{t=0}=q_{0} \in \mathcal{D}_{\omega}^{\Delta, \sigma} .
$$

As above, one can show that $\mathcal{G}_{\omega^{\prime}} \subset \mathcal{D}_{\omega}^{\Delta, \sigma}$ for each $\omega^{\prime}>\omega$. By (5.17) and (4.2) for $u \in \mathcal{U}_{\sigma, \alpha}$ we have

$$
\begin{aligned}
|u|_{\omega} & \leq\|u\|_{\sigma, \alpha} \int_{\Gamma_{0}} \exp ((\omega+\alpha)|\eta|) e\left(\varphi_{\sigma} ; \eta\right) \lambda(d \eta) \\
& \leq\|u\|_{\sigma, \alpha} \exp \left(\bar{\varphi}_{\sigma} e^{\omega+\alpha}\right),
\end{aligned}
$$

see also (5.8). Hence $\mathcal{U}_{\sigma, \alpha} \hookrightarrow \mathcal{G}_{\omega}$ for each $\omega$ and $\alpha$. Like in (5.20) we then get

$$
\left(L_{\alpha, u}^{\Delta, \sigma}, \mathcal{D}_{\alpha, u}^{\Delta, \sigma}\right) \subset\left(L_{\omega}^{\Delta, \sigma}, \mathcal{D}_{\omega}^{\Delta, \sigma}\right) .
$$

Lemma 5.5 Assume that the problem (5.25) with $\omega>0$ and $q_{0} \in \mathcal{G}_{\omega^{\prime}}$, $\omega^{\prime}>\omega$, has a solution, $q_{t} \in \mathcal{G}_{\omega}$, on some time interval $\left[0, T\left(\omega^{\prime}, \omega\right)\right)$. Then this solution is unique.

Proof Set

$$
w_{t}(\eta)=(-1)^{|\eta|} q_{t}(\eta),
$$

which is an isometry on $\mathcal{G}_{\omega}$. Then $q_{t}$ solves (5.25) if and only if $w_{t}$ solves the following equation

$$
\begin{aligned}
\frac{d}{d t} w_{t}(\eta)= & -E(\eta) w_{t}(\eta)+\int_{\mathbb{R}^{d}} E^{-}(y, \eta) w_{t}(\eta \cup y) d y \\
& -\sum_{x \in \eta} \varphi_{\sigma}(x) E^{+}(x, \eta \backslash x) w_{t}(\eta \backslash x) \\
& +\int_{\mathbb{R}^{d}} \sum_{x \in \eta} \varphi_{\sigma}(x) a^{+}(x-y) w_{t}(x \backslash x \cup y) d y .
\end{aligned}
$$

Set

$$
\mathcal{D}_{\omega}=\left\{w \in \mathcal{G}_{\omega}: E(\cdot) w \in \mathcal{G}_{\omega}\right\} .
$$

By Proposition 4.1 we prove that the operator defined by the first two summands in (5.28) with domain $\mathcal{D}_{\omega}$ generates a substochastic semigroup, $\left\{V_{\omega}(t)\right\}_{t \geq 0}$, acting in $\mathcal{G}_{\omega}$. Indeed, in this case the condition analogous to that in (4.8) takes the form, cf. (4.9),

$$
\begin{aligned}
& -\int_{\Gamma_{0}} E(\eta) w(\eta) \exp (\omega|\eta|) \lambda(d \eta) \\
& +r^{-1} e^{-\omega} \int_{\Gamma_{0}} E^{-}(\eta) w(\eta) \exp (\omega|\eta|) \lambda(d \eta) \leq 0,
\end{aligned}
$$


which certainly holds for each $\omega>0$ and an appropriate $r<1$. For each $\omega^{\prime \prime} \in(0, \omega)$, we have that $\mathcal{G}_{\omega} \hookrightarrow \mathcal{G}_{\omega^{\prime \prime}}$, and the second two summands in (5.28) define a bounded operator, $W_{\omega^{\prime \prime} \omega}: \mathcal{G}_{\omega} \rightarrow \mathcal{G}_{\omega^{\prime \prime}}$, the norm of which can be estimated as follows, cf. (5.3),

$$
\left\|W_{\omega^{\prime \prime} \omega}\right\| \leq \frac{\left(e^{\omega}+1\right)\left\langle a^{+}\right\rangle}{e\left(\omega-\omega^{\prime \prime}\right)} .
$$

Assume now that (5.28) has two solutions corresponding to the same initial condition $w_{0}(\eta)=$ $(-1)^{|\eta|} q_{0}(\eta)$. Let $v_{t}$ be their difference. Then it solves the following equation, cf. (4.38),

$$
v_{t}=\int_{0}^{t} V_{\omega^{\prime \prime}}(t-s) W_{\omega^{\prime \prime} \omega} v_{s} d s,
$$

where $v_{t}$ on the left-hand side is considered as an element of $\mathcal{G}_{\omega^{\prime \prime}}$ and $t>0$ will be chosen later. Now for a given $n \in \mathbb{N}$, we set $\epsilon=\left(\omega-\omega^{\prime \prime}\right) / n$ and then $\omega^{l}:=\omega-l \epsilon, l=0, \ldots, n$. Thereafter, we iterate (5.30) and get

$$
\begin{aligned}
v_{t}= & \int_{0}^{t} \int_{0}^{t_{1}} \cdots \int_{0}^{t_{n-1}} V_{\omega^{\prime \prime}}\left(t-t_{1}\right) W_{\omega^{\prime \prime} \omega^{n-1}} V_{\omega^{n-1}}\left(t_{1}-t_{2}\right) \times \cdots \\
& \times W_{\omega^{2} \omega^{1}} V_{\omega^{1}}\left(t_{n-1}-t_{n}\right) W_{\omega^{1} \omega} v_{t_{n}} d t_{n} \cdots d t_{1} .
\end{aligned}
$$

Similarly as in (4.39), by (5.29) this yields the following estimate

$$
\left|v_{t}\right|_{\omega^{\prime \prime}} \leq \frac{1}{n !}\left(\frac{n}{e}\right)^{n}\left(\frac{t\left\langle a^{+}\right\rangle\left(e^{\omega}+1\right)}{\omega-\omega^{\prime \prime}}\right)^{n} \sup _{s \in[0, t]}\left|v_{s}\right|_{\omega} .
$$

The latter implies that $v_{t}=0$ for $t<\left(\omega-\omega^{\prime \prime}\right) /\left\langle a^{+}\right\rangle\left(e^{\omega}+1\right)$. To prove that $v_{t}=0$ for all $t$ of interest one has to repeat the above procedure appropriate number of times.

Recall that each $\mathcal{U}_{\sigma, \alpha}$ is continuously embedded into each $\mathcal{G}_{\omega}$, see (5.26).

Corollary 5.6 For each $\omega>0$, the problem (5.25) with $q_{0} \in \mathcal{U}_{\sigma, \alpha_{0}}$ has a unique solution $q_{t}$ which coincides with the solution $u_{t} \in \mathcal{U}_{\sigma, \alpha}$ mentioned in Lemma 5.3.

Proof By (5.27) $u_{t}$ is a solution of (5.25). Its uniqueness follows by Lemma 5.5.

\subsection{Local Evolution}

In this subsection we pass to the so called local evolution of states of the auxiliary model (5.10), (5.11). For this evolution, the corresponding 'correlation function' $q_{t} \in \mathcal{G}_{\omega}$ has the positive definiteness in question. Then we apply Corollaries 5.4 and 5.6 to get the same for the evolution in $\mathcal{K}_{\alpha}$. Thereafter, we pass to the limit and get the proof of Lemma 4.9.

\subsubsection{The Evolution of Densities}

In view of (2.2), each state with the property $\mu\left(\Gamma_{0}\right)=1$ can be redefined as a probability measure on $\mathcal{B}\left(\Gamma_{0}\right)$, cf. Remark 2.1. Then the Fokker-Planck equation (1.3) can be studied directly, see [23, Eq. (2.8)]. Its solvability is described in [23, Theorem 2.2], which, in particular, states that the solution is absolutely continuous with respect to the LebesguePoisson measure $\lambda$ if $\mu_{0}$ has the same property. In view of this we write the corresponding problem for the density

$$
R_{t}:=\frac{d \mu_{t}}{d \lambda},
$$


see also [23, Eq. (2.16)], and obtain

$$
\frac{d}{d t} R_{t}(\eta)=\left(L^{\dagger, \sigma} R_{t}\right)(\eta),\left.\quad R_{t}\right|_{t=0}=R_{0},
$$

where

$$
\begin{aligned}
\left(L^{\dagger, \sigma} R\right)(\eta) & :=-\Psi_{\sigma}(\eta) R(\eta)+\sum_{x \in \eta} \varphi_{\sigma}(x) E^{+}(x, \eta \backslash x) R_{t}(\eta \backslash x) \\
& +\int_{\mathbb{R}^{d}}\left(m+E^{-}(x, \eta)\right) R_{t}(\eta \cup x) d x,
\end{aligned}
$$

and

$$
\Psi_{\sigma}(\eta)=E(\eta)+\int_{\mathbb{R}^{d}} \varphi_{\sigma}(x) E^{+}(x, \eta) d x .
$$

We solve (5.32) in the Banach spaces $\mathcal{G}_{0}=L^{1}\left(\Gamma_{0}, d \lambda\right)$, cf. (4.1). For $n \in \mathbb{N}$ we denote by $\mathcal{G}_{0, n}$ the subset of $\mathcal{G}_{0}$ consisting of all those $R: \Gamma_{0} \rightarrow \mathbb{R}$ for which

$$
\int_{\mathbb{R}^{d}}|\eta|^{n}|R(\eta)| \lambda(d \eta)<\infty
$$

Let also $\mathcal{G}_{\omega}^{+}$stand for the cone of positive elements of $\mathcal{G}_{\omega}$. Set

$$
\mathcal{D}_{0}=\left\{R \in \mathcal{G}_{0}: \Psi_{\sigma} R \in \mathcal{G}_{0}\right\} .
$$

Then the relevant part of [23, Theorem 2.2] can be formulated as follows.

Proposition 5.7 The closure in $\mathcal{G}_{0}$ of the operator $\left(L^{\dagger, \sigma}, \mathcal{D}_{0}\right)$ defined in (5.33) and (5.34) generates a stochastic semigroup $\left\{S^{\dagger, \sigma}(t)\right\}_{t \geq 0}:=S^{\dagger, \sigma}$ of bounded operators in $\mathcal{G}_{0}$, which leaves invariant each $\mathcal{G}_{0, n}, n \in \mathbb{N}$. Moreover, for each $\beta^{\prime}>0$ and $\beta \in\left(0, \beta^{\prime}\right), R \in \mathcal{G}_{\beta^{\prime}}^{+}$ implies $S^{\dagger, \sigma}(t) R \in \mathcal{G}_{\beta}^{+}$holding for all $t<T\left(\beta^{\prime}, \beta\right)$, where $T\left(\beta^{\prime}, \beta\right)=+\infty$ for $\left\langle a^{+}\right\rangle=0$, and

$$
T\left(\beta^{\prime}, \beta\right)=\left(\beta^{\prime}-\beta\right) e^{-\beta^{\prime}} /\left\langle a^{+}\right\rangle, \quad \text { for }\left\langle a^{+}\right\rangle>0 .
$$

Let now $\mu_{0}$ be the initial state as in Theorem 3.3. Then for each $\Lambda \in \mathcal{B}_{\mathrm{b}}\left(\mathbb{R}^{d}\right)$, the projection $\mu^{\Lambda}$ is absolutely continuous with respect to $\lambda^{\Lambda}$, see (2.7). For this $\mu_{0}$, and for $\Lambda \in \mathcal{B}_{\mathrm{b}}\left(\mathbb{R}^{d}\right)$ and $N \in \mathbb{N}$, we set, see (5.31),

$$
R_{0}^{\Lambda}(\eta)=\frac{d \mu^{\Lambda}}{d \lambda^{\Lambda}}(\eta) \mathbb{I}_{\Gamma_{\Lambda}}(\eta), \quad R_{0}^{\Lambda, N}(\eta)=R_{0}^{\Lambda}(\eta) I_{N}(\eta), \quad \eta \in \Gamma_{0} .
$$

Here $I_{N}$ and $\mathbb{I}_{\Gamma_{\Lambda}}$ are the indicator functions of the sets $\left\{\eta \in \Gamma_{0}:|\eta| \leq N\right\}, N \in \mathbb{N}$, and $\Gamma_{\Lambda}$, respectively. Clearly,

$$
\forall \beta>0 \quad R_{0}^{\Lambda, N} \in \mathcal{G}_{\beta}^{+} .
$$

Set

$$
R_{t}^{\Lambda, N}=S^{\dagger, \sigma}(t) R_{0}^{\Lambda, N}, \quad t>0,
$$

where $S^{\dagger, \sigma}$ is the semigroup as in Proposition 5.7. Then also $R_{t}^{\Lambda, N} \in \mathcal{G}_{0}^{+}$for all $t>0$.

For some $G \in B_{\mathrm{bs}}\left(\Gamma_{0}\right)$, let us consider $F=K G$, cf. (2.4). Since $G(\xi)=0$ for all $\xi$ such that $|\xi|>N(G)$, see Definition 2.2, we have $F \in \mathcal{F}_{\text {cyl }}(\Gamma)$ and

$$
|F(\gamma)| \leq(1+|\gamma|)^{N(G)} C(G), \quad \gamma \in \Gamma_{0},
$$

for some $C(G)>0$. By Proposition 5.7 we then have from the latter

$$
\left|\left\|K G, R_{t}^{\Lambda, N}\right\|\right|<\infty \text {. }
$$




\subsubsection{The Evolution of Local Correlation Functions}

For a given $\mu \in \mathcal{P}_{\mathrm{sP}}$, the correlation function $k_{\mu}$ and the local densities $R_{\mu}^{\Lambda}, \Lambda \in \mathcal{B}_{\mathrm{b}}\left(\mathbb{R}^{d}\right)$, see (2.8), are related to each other by (2.9). In the first formula of (5.36) we extend $R_{0}^{\Lambda}$ to the whole $\Gamma_{0}$. Then the corresponding integral as in (2.9) coincides with $k_{\mu_{0}}$ only on $\Gamma_{\Lambda}$. The truncation made in the second formula in (5.36) diminishes $R_{0}^{\Lambda}$. Its aim is to satisfy (5.37). Thus, with a certain abuse of the terminology we call

$$
q_{0}^{\Lambda, N}(\eta)=\int_{\Gamma_{0}} R_{0}^{\Lambda, N}(\eta \cup \xi) \lambda(d \xi)
$$

local correlation function. The evolution $q_{0}^{\Lambda, N} \mapsto q_{t}^{\Lambda, N}$ can be obtained from (5.38) by setting

$$
q_{t}^{\Lambda, N}(\eta)=\int_{\Gamma_{0}} R_{t}^{\Lambda, N}(\eta \cup \xi) \lambda(d \xi), \quad t \geq 0 .
$$

However, so far this can only be used in a weak sense based on (5.39). Note that for $G \in$ $B_{\mathrm{bs}}^{\star}\left(\Gamma_{0}\right)$, cf. (2.11), we have

$$
\left\|G, q_{t}^{\Lambda, N} \rrbracket=\right\| K G, R_{t}^{\Lambda, N} \rrbracket \geq 0,
$$

since $R_{t}^{\Lambda, N} \in \mathcal{G}_{0}^{+}$. To place the evolution $q_{0}^{\Lambda, N} \mapsto q_{t}^{\Lambda, N}$ into an appropriate Banach space we use the concluding part of Proposition 5.7 and the following fact

$$
\int_{\Gamma_{0}} e^{\omega|\eta|} q_{t}^{\Lambda, N}(\eta) \lambda(d \eta)=\int_{\Gamma_{0}}\left(1+e^{\omega}\right)^{|\eta|} R_{t}^{\Lambda, N}(\eta) \lambda(d \eta),
$$

that can be obtained by (2.13). Since $R_{0}^{\Lambda, N} \in \mathcal{G}_{\beta^{\prime}}$ for any $\beta^{\prime}>0$, see (5.37), we can take $\beta^{\prime}=\beta+1$ which maximizes $T\left(\beta^{\prime}, \beta\right)$ given in (5.35). Then for each $\beta>0$, we have that

$$
R_{t}^{\Lambda, N} \in \mathcal{G}_{\beta}, \quad \text { for } t<\tau(\beta):=\frac{e^{-\beta}}{e\left\langle a^{+}\right\rangle} .
$$

Hence, $q_{t}^{\Lambda, N} \in \mathcal{G}_{\omega}$ whenever $R_{t}^{\Lambda, N} \in \mathcal{G}_{\beta}$ with $\beta$ such that $e^{\beta}=1+e^{\omega}$, cf. (5.43). Moreover, for such $\omega$ and $\beta$ the right-hand side of (5.41) defines a continuous map from $\mathcal{G}_{\beta}$ to $\mathcal{G}_{\omega}$.

Lemma 5.8 Given $\omega_{1}>0$ and $\omega_{2}>\omega_{1}$, let $\beta_{i}$ be such that $e^{\beta_{i}}=e^{\omega_{i}}+1, i=1,2$. Then $q_{t}^{\Lambda, N}$ is continuously differentiable in $\mathcal{G}_{\omega_{1}}$ on $\left[0, \tau\left(\beta_{2}\right)\right)$ and the following holds

$$
\frac{d}{d t} q_{t}^{\Lambda, N}=L_{\omega_{1}}^{\Delta, \sigma} q_{t}^{\Lambda, N}
$$

Proof By the mentioned continuity of the map in (5.41) the continuous differentiability of $q_{t}^{\Lambda, N}$ follows from the corresponding property of $R_{t}^{\Lambda, N} \in \mathcal{G}_{\beta_{2}}$, which it has in view of (5.38). Then the following holds

$$
\left(\frac{d}{d t} q_{t}^{\Lambda, N}\right)(\eta)=\int_{\Gamma_{0}}\left(L_{\beta_{1}}^{\dagger, \sigma} R_{t}^{\Lambda, N}\right)(\eta \cup \xi) \lambda(d \xi)
$$

Where $L_{\beta_{1}}^{\dagger, \sigma}$ is the trace of $L^{\dagger, \sigma}$ in $\mathcal{G}_{\beta_{1}}$. We define the action of $\widehat{L}^{\sigma}=A^{\sigma}+B^{\sigma}$ in such a way that

$$
\| \widehat{L}^{\sigma} G, k \rrbracket=\left\langle G, L^{\Delta, \sigma} k\right\rangle,
$$


where that $L^{\Delta, \sigma}$ acts as in (5.10) and (5.11). Then $A^{\sigma}$ acts as in (4.5) with $E^{+}(y, \eta)$ replaced by $\varphi_{\sigma}(y) E^{+}(y, \eta)$, and $B^{\sigma}$ acts as in (5.2) with $a^{+}(x-y)$ multiplied by $\varphi_{\sigma}(x)$. Let $G$ : $\Gamma_{0} \rightarrow \mathbb{R}$ be bounded and continuous. Then for some $C>0$ we have, see (2.4),

$$
\left|\widehat{L}^{\sigma} G(\eta)\right| \leq|\eta|^{2} C \sup _{\eta \in \Gamma_{0}}|G(\eta)|, \quad\left|K\left(\widehat{L}^{\sigma} G\right)(\eta)\right| \leq|\eta|^{2} 2^{|\eta|} C \sup _{\eta \in \Gamma_{0}}|G(\eta)|,
$$

and hence we can calculate the integrals below

$$
\left.\| \widehat{L}^{\sigma} G, q_{t}^{\Lambda, N}\right\rangle=\| G, L_{\omega_{1}}^{\Delta, \sigma} q_{t}^{\Lambda, N} \rrbracket,
$$

where $\omega_{1}$ and $q_{t}^{\Lambda, N}$ are as in (5.46). On the other hand, by (5.46) we have

$$
\begin{aligned}
\left\langle\widehat{L}^{\sigma} G, q_{t}^{\Lambda, N}\right\rangle & \left.=\| K \widehat{L}^{\sigma} G, R_{t}^{\Lambda, N}\right\rangle \\
& \left.=\| K G, L_{\beta_{1}}^{\dagger, \sigma} R_{t}^{\Lambda, N}\right\rangle=\| G, \frac{d}{d t} q_{t}^{\Lambda, N} \rrbracket .
\end{aligned}
$$

Since (5.47) and (5.48) hold true for all bounded continuous functions, we have that the expression on both sides of (5.45) are equal to each other, which completes the proof.

Corollary 5.9 Let $k_{t}^{\Lambda, N} \in \mathcal{K}_{\alpha_{2}}$ be the solution of the problem (5.14) with $k_{0}^{\Lambda, N}=q_{0}^{\Lambda, N} \in$ $\mathcal{K}_{\alpha_{1}}$, see Lemma 5.2. Then for each $G \in B_{\mathrm{bs}}^{\star}\left(\Gamma_{0}\right)$ and

$$
t<\min \left\{T\left(\alpha_{2}, \alpha_{1} ; B^{\Delta}\right) ; 1 / e\left\langle a^{+}\right\rangle\right\}
$$

see (5.44), we have that

$$
\| G, k_{t}^{\Lambda, N} \rrbracket \geq 0
$$

Proof By (5.36) and (5.40) we have that $q_{0}^{\Lambda, N} \in \mathcal{U}_{\sigma, \alpha_{1}}$ (this is the reason to consider such local evolutions). Let then $u_{t}$ be the solution as in Lemma 5.3 with this initial condition. Then by Corollaries 5.4 and 5.6 it follows that $k_{t}^{\Lambda, N}=u_{t}=q_{t}^{\Lambda, N}$ for the mentioned values of $t$. Thus, the validity of (5.49) follows by (5.42).

\subsection{Taking the Limits}

Note that (5.49) holds for

$$
k_{t}^{\Lambda, N}=Q_{\alpha \alpha_{1}}^{\sigma}\left(t ; B_{b}^{\Delta, \sigma}\right) q_{0}^{\Lambda, N}
$$

with $\alpha \in\left(\alpha_{1}, \alpha_{2}\right)$ dependent on $t$, see (5.16). In this subsection, we first pass in (5.49) to the limit $\sigma \downarrow 0$, then we get rid of the locality imposed in (5.36).

Lemma 5.10 Let $k_{t}$ and $k_{t}^{\sigma}$ be the solutions of the problems (3.17) and (5.14), respectively, with $\left.k_{t}\right|_{t=0}=\left.k_{t}^{\sigma}\right|_{t=0}=k_{0} \in \mathcal{K}_{\alpha_{0}}, \alpha_{0}>-\log \vartheta$. Then for each $\alpha>\alpha_{0}$ there exists $\widetilde{T}=\widetilde{T}\left(\alpha, \alpha_{0}\right)<T\left(\alpha, \alpha_{0} ; B_{b}^{\Delta}\right)$ such that for each $G \in B_{\mathrm{bs}}\left(\Gamma_{0}\right)$ and $t \in[0, \widetilde{T}]$ the following holds

$$
\lim _{\sigma \downarrow 0}\left\langle\left\langle G, k_{t}^{\sigma}\right\rangle\right\rangle=\left\langle\left\langle G, k_{t}\right\rangle\right\rangle
$$

Proof Take $\alpha_{2} \in\left(\alpha_{0}, \alpha\right)$ and $\alpha_{1} \in\left(\alpha_{0}, \alpha_{2}\right)$. Thereafter, take

$$
\widetilde{T}<\min \left\{T\left(\alpha_{1}, \alpha_{0} ; B_{b}^{\Delta}\right) ; T\left(\alpha, \alpha_{2} ; B_{b}^{\Delta}\right)\right\} .
$$


For $t \leq \widetilde{T}$, by (4.27), (4.37), (5.11), and (5.16) we then have that the following holds, see (4.37) and (5.24),

$$
\begin{aligned}
Q_{\alpha \alpha_{0}}\left(t ; B_{b}^{\Delta}\right) k_{0} & =Q_{\alpha \alpha_{0}}^{\sigma}(t) k_{0}+M_{\sigma}(t)+N_{\sigma}(t) \\
M_{\sigma}(t) & :=\int_{0}^{t} Q_{\alpha \alpha_{2}}\left(t-s ; B_{b}^{\Delta}\right)\left(\left(A_{2}^{\Delta}\right)_{\alpha_{2} \alpha_{1}}-\left(A_{2}^{\Delta, \sigma}\right)_{\alpha_{2} \alpha_{1}}\right) k_{s}^{\sigma} d s \\
N_{\sigma}(t) & :=\int_{0}^{t} Q_{\alpha \alpha_{2}}\left(t-s ; B_{b}^{\Delta}\right)\left(\left(B_{2, b}^{\Delta}\right)_{\alpha_{2} \alpha_{1}}-\left(B_{2, b}^{\Delta, \sigma}\right)_{\alpha_{2} \alpha_{1}}\right) k_{s}^{\sigma} d s
\end{aligned}
$$

where

$$
k_{s}^{\sigma}=Q_{\alpha_{1} \alpha_{0}}^{\sigma}\left(s ; B_{b}^{\Delta}\right) k_{0}
$$

Then

$$
\left\langle\left\langle G, k_{t}\right\rangle\right\rangle-\left\langle\left\langle G, k_{t}^{\sigma}\right\rangle\right\rangle=\left\langle\left\langle G, M_{\sigma}(t)\right\rangle\right\rangle+\left\langle\left\langle G, N_{\sigma}(t)\right\rangle\right\rangle .
$$

By (5.5) we get

$$
\begin{aligned}
\left\langle\left\langle G, M_{\sigma}(t)\right\rangle\right\rangle & =\int_{0}^{t}\left\langle G, Q_{\alpha \alpha_{2}}\left(t-s ; B_{b}^{\Delta}\right) v_{s}\right\rangle d s \\
& =\int_{0}^{t}\left\langle\left\langle H_{\alpha_{2} \alpha}(t-s) G, v_{s}\right\rangle\right\rangle d s=\int_{0}^{t}\left\langle\left\langle G_{t-s}, v_{s}\right\rangle\right\rangle d s,
\end{aligned}
$$

where

$$
\begin{aligned}
& \left\langle\left\langle G_{t-s}, v_{s}\right\rangle\right\rangle \\
& =\int_{\Gamma_{0}} G_{t-s}(\eta) \sum_{x \in \eta}\left(1-\varphi_{\sigma}(x)\right) E^{+}(x, \eta \backslash x) k_{s}^{\sigma}(\eta \backslash x) \lambda(d \eta) \\
& =\int_{\Gamma_{0}} \int_{\mathbb{R}^{d}} G_{t-s}(\eta \cup x)\left(1-\varphi_{\sigma}(x)\right) E^{+}(x, \eta) k_{s}^{\sigma}(\eta) d x \lambda(d \eta),
\end{aligned}
$$

where the latter line was obtained by means of (2.13). Note that $k_{s}^{\sigma} \in \mathcal{K}_{\alpha_{1}}$ and $G_{t-s} \in \mathcal{G}_{\alpha_{2}}$ for $s \leq t \leq \widetilde{T}$, see (5.51). We use this fact to prove that

$$
g_{s}(x):=\int_{\Gamma_{0}} \frac{1}{|\eta|+1}\left|G_{s}(\eta \cup x)\right| e^{\alpha_{2}|\eta|} \lambda(d \eta)
$$

lies in $L^{1}\left(\mathbb{R}^{d}\right)$ for each $s \in[0, t]$. Indeed, by (2.13) and (4.2) we get

$$
\left\|g_{s}\right\|_{L^{1}\left(\mathbb{R}^{d}\right)} \leq e^{-\alpha_{2}}\left|G_{s}\right|_{\alpha_{2}} \leq C_{1}<\infty
$$

where

$$
C_{1}:=e^{-\alpha_{2}} \max _{s \in[0, \widetilde{T}]}\left|G_{s}\right|_{\alpha_{2}} \leq \frac{e^{-\alpha_{0}} T\left(\alpha, \alpha_{2} ; B_{b}^{\Delta}\right)|G|_{\alpha}}{T\left(\alpha, \alpha_{2} ; B_{b}^{\Delta}\right)-\widetilde{T}},
$$

see (5.6). By (5.15) and (5.52) we also get

$$
\max _{s \in[0, \widetilde{T}]}\left\|k_{s}^{\sigma}\right\|_{\alpha_{2}} \leq \frac{T\left(\alpha_{1}, \alpha_{0} ; B_{b}^{\Delta}\right)\left\|k_{0}\right\|_{\alpha_{0}}}{T\left(\alpha_{1}, \alpha_{0} ; B_{b}^{\Delta}\right)-\widetilde{T}}=: C_{2}<\infty
$$


see (5.51). Now we use (5.55), (5.56), (5.58) and obtain by (3.2) and (3.12) that the following holds

$$
\begin{aligned}
\left|\left\langle\left\langle G, M_{\sigma}(t)\right\rangle\right\rangle\right| & \leq \varkappa\left(\alpha_{2}-\alpha_{1}\right)\left\|a^{+}\right\| e^{\alpha_{1}} C_{2} \\
& \times \int_{0}^{\widetilde{T}} \int_{\mathbb{R}^{d}} g_{s}(x)\left(1-\varphi_{\sigma}(x)\right) d x d s,
\end{aligned}
$$

where

$$
\varkappa(\beta):=\frac{1}{e \beta}+\left(\frac{2}{e \beta}\right)^{2} .
$$

By (5.56) and (5.57) we conclude that the integrand in the right-hand side of (5.59) is bounded by $C_{1}$. By the Lebesgue dominated convergence theorem this yields RHS (5.59) $\rightarrow 0$ as $\sigma \downarrow 0$. In the same way one proves that also

$$
\left|\left\langle\mid G, N_{\sigma}(t)\right\rangle\right\rangle \mid \rightarrow 0, \quad \sigma \downarrow 0,
$$

which yields (5.50), see (5.53).

Below by a cofinal sequence $\left\{\Lambda_{n}\right\}_{n \in \mathbb{N}} \subset \mathcal{B}_{\mathrm{b}}\left(\mathbb{R}^{d}\right)$ we mean a sequence such that: $\Lambda_{n} \subset \Lambda_{n+1}$ for all $n$, and each $x \in \mathbb{R}$ belongs to a certain $\Lambda_{n}$.

Lemma 5.11 Let $\left\{\Lambda_{n}\right\}_{n \in \mathbb{N}}$ be a cofinal sequence and $q_{0}^{\Lambda, N}$ be as in (5.40). Let also $\alpha_{1}$ and $\alpha_{2}$ be as in Lemma 4.9. Then for each $t \in\left[0, T\left(\alpha_{2}, \alpha_{1} ; B_{b}^{\Delta}\right)\right)$ and $G \in B_{\mathrm{bs}}\left(\Gamma_{0}\right)$, the following holds

$$
\lim _{n \rightarrow+\infty} \lim _{N \rightarrow+\infty}\left\|G, Q_{\alpha_{2} \alpha_{1}}\left(t ; B_{b}^{\Delta}\right) q_{0}^{\Lambda_{n}, N} \rrbracket=\right\| G, Q_{\alpha_{2} \alpha_{1}}\left(t ; B_{b}^{\Delta}\right) k_{\mu_{0}} \rrbracket .
$$

Proof As in (5.54), we prove (5.60) by showing that

$$
\begin{aligned}
& \lim _{n \rightarrow+\infty} \lim _{N \rightarrow+\infty}\left\|G, Q_{\alpha_{2} \alpha_{1}}\left(t ; B_{b}^{\Delta}\right) q_{0}^{\Lambda_{n}, N}\right\| \\
= & \left.\lim _{n \rightarrow+\infty} \lim _{N \rightarrow+\infty} \| H_{\alpha_{1} \alpha_{2}}(t) G, q_{0}^{\Lambda_{n}, N}\right\rangle=\left\langle H_{\alpha_{1} \alpha_{2}}(t) G, k_{\mu_{0}} \| .\right.
\end{aligned}
$$

Since $G_{t}:=H_{\alpha_{1} \alpha_{2}}(t) G$ lies in $\mathcal{G}_{\alpha_{1}}$, the proof of (5.61) can be done by the repetition of arguments used in the proof of the analogous result in [5, Appendix].

\subsection{The Proof of Lemma 4.9}

Let $\alpha_{1}$ and $\alpha_{2}$ be as in Lemma 4.9 and $\left\{\Lambda_{n}\right\}_{n \in \mathbb{N}}$ be a cofinal sequence. Take $k_{\mu_{0}} \in \mathcal{K}_{\alpha_{1}}$ and then produce $q_{0}^{\Lambda_{n}, N}, n \in \mathbb{N}$, by employing (5.36) and (5.40). Let $T\left(\alpha_{2}, \alpha_{1}\right)<T\left(\alpha_{2}, \alpha_{1} ; B_{b}^{\Delta}\right)$ be such that (5.49) holds with

$$
k_{t}^{\Lambda_{n}, N}=Q^{\sigma}\left(\alpha_{2}, \alpha_{1} ; B_{b}^{\Delta}\right) q_{0}^{\Lambda_{n}, N}, \quad t \leq T\left(\alpha_{2}, \alpha_{1}\right) .
$$

Note that $T\left(\alpha_{2}, \alpha_{1}\right)$ is independent of $\Lambda_{n}$ and $N$, see Corollary 5.9. By Lemma 5.11 we then have that

$$
\| G, Q_{\alpha_{2} \alpha_{1}}^{\sigma}\left(t ; B_{b}^{\Delta}\right) k_{\mu_{0}} \rrbracket \geq 0 .
$$

Now we apply Lemma 5.10 and obtain

$$
\| G, Q_{\alpha_{2} \alpha_{1}}\left(t ; B_{b}^{\Delta}\right) k_{\mu_{0}} \rrbracket \geq 0,
$$


which holds for

$$
t \leq \tau\left(\alpha_{2}, \alpha_{1}\right):=\min \left\{T\left(\alpha_{2}, \alpha_{1}\right) ; \widetilde{T}\left(\alpha_{2}, \alpha_{1}\right)\right\},
$$

which completes the proof.

Acknowledgements This paper was partially supported by the DFG through the SFB 701 "Spektrale Strukturen und Topologische Methoden in der Mathematik" and by the European Commission under the Project STREVCOMS PIRSES-2013-612669.

Open Access This article is distributed under the terms of the Creative Commons Attribution 4.0 International License (http://creativecommons.org/licenses/by/4.0/), which permits unrestricted use, distribution, and reproduction in any medium, provided you give appropriate credit to the original author(s) and the source, provide a link to the Creative Commons license, and indicate if changes were made.

\section{References}

1. Albeverio, S., Kondratiev, Y.G., Röckner, M.: Analysis and geometry on configuration spaces. J. Funct. Anal. 154, 444-500 (1998)

2. Angelescu, N., Nenciu, G., Protopopescu, V.: On stable potentials. Commun. Math. Phys. 22, 162-165 (1971)

3. Banasiak, J., Lachowicz, M., Moszyński, M.: Semigroups for generalized birth-and-death equations in $\ell^{p}$ spaces. Semigroup Forum 73, 175-193 (2006)

4. Bellomo, N., Brezzi, F.: Mathematics, complexity and multiscale features of large systems of selfpropelled particles. Math. Models Methods Appl. Sci. 26, 207-214 (2016)

5. Berns, C.H., Kondratiev, Y., Kozitsky, Y., Kutovyi, O.: Kawasaki dynamics in continuum: micro- and mesoscopic descriptions. J. Dyn. Differ. Equ. 25, 1027-1056 (2013)

6. Bolker, B.M., Pacala, S.W.: Using moment equations to understand stochastically driven spatial pattern formation in ecological systems. Theor. Popul. Biol. 52, 179-197 (1997)

7. Bolker, B.M., Pacala, S.W., Neuhauser, C.: Spatial dynamics in model plant communities: what do we really know? Am. Nat. 162, 135-148 (2003)

8. Conway, J.H., Sloan, N.J.A.: Sphere packing, lattices and groups. In: Bannai, E., Borcherds, R.E., Leech, J., Norton, S.P., Odlyzko, A.M., Parker, R.A., Queen, L., Venkov, B.B. (eds.) Grundlehren der Mathematischen Wissenschaften, 3rd edn. Springer, New York (1999)

9. Feller, W.: An Introduction to Probability Theory and its Applications, 3rd edn. Wiley, New York (1970)

10. Finkelshtein, D.L., Kondratiev, Y.G., Kutovyi, O.: Individual based model with competition in spatial ecology. SIAM J. Math. Anal. 41, 297-317 (2009)

11. Finkelshtein, D.L., Kondratiev, Y.G., Oliveira, M.J.: Markov evolutions and hierarchical equations in the continuum. I. One-component systems. J. Evol. Equ. 9, 197-233 (2009)

12. Finkelshtein, D.L., Kondratiev, Y.G., Kutovyi, O.: Semigroup approach to birth-and-death stochastic dynamics in continuum. J. Funct. Anal. 262, 1274-1308 (2012)

13. Finkelshtein, D.L., Kondratiev, Y.G., Kozitsky, Y., Kutovyi, O.: The statistical dynamics of a spatial logistic model and the related kinetic equation. Math. Models Methods Appl. Sci. 25, 343-370 (2015)

14. Garcia, N.L., Kurtz, T.G.: Spatial birth and death processes as solutions of stochastic equations, ALEA Lat. Am. J. Probab. Math. Stat. 1, 281-303 (2006)

15. Holley, R.A., Stroock, D.W.: Nearest neighbor birth and death processes on the real line. Acta Math. 140, 103-154 (1978)

16. Kijima, M.: Markov Processes for Stochastic Modelling, Stochastic Modeling Series. Chapman \& Hall, London (1997)

17. Kondratiev, Y., Kuna, T.: Harmonic analysis on configuration space. I. General theory. Infin. Dimens. Anal. Quantum Probab. Relat. Top. 5, 201-233 (2002)

18. Kondratiev, Y.G., Skorokhod, A.V.: On contact models in continuum. Infin. Dimens. Anal. Quantum Probab. Relat. Top. 9, 187-198 (2006)

19. Kondratiev, Y., Kuna, T., Oliveira, M.J.: Holomorphic Bogoliubov functionals for interacting particle systems in continuum. J. Funct. Anal. 238, 375-404 (2006)

20. Kondratiev, Y., Kutoviy, O., Minlos, R.: On non-equilibrium stochastic dynamics for interacting particle systems in continuum. J. Funct. Anal. 255, 200-227 (2008) 
21. Kondratiev, Y., Kutovyi, O., Pirogov, S.: Correlation functions and invariant measures in continuous contact model. Infin. Dimens. Anal. Quantum Probab. Relat. Top. 11, 231-258 (2008)

22. Kondratiev, Y., Kozitsky, Y., Shoikhet, D.: Dynamical systems on sets of holomorphic functions. Contemp. Math. 553, 139-153 (2011)

23. Kozitsky, Y.: Dynamics of spatial logistic model: finite systems. In: Banasiak, J., Bobrowski, A., Lachowicz, M. (eds.), Semigroups of Operators-Theory and Applications: Bȩdlewo, Poland, October 2013. Springer Proceedings in Mathematics \& Statistics, vol. 113, pp. 197-211. Springer (2015)

24. Murrell, D.J., Dieckmann, U., Law, R.: On moment closures for population dynamics in contunuous space. J. Theor. Biol. 229, 421-432 (2004)

25. Neuhauser, C.: Mathematical challenges in spatial ecology. Not. AMS 48(11), 1304-1314 (2001)

26. Obata, N.: Configuration space and unitary representations of the group of diffeomorphisms. RIMS Kôkyûroku 615, 129-153 (1987)

27. Ovaskainen, O., Finkelshtein, D., Kutovyi, O., Kornel, S., Bolker, B., Kondratiev, Y.: A general mathematical framwork for the analysis of spatio-temporal point processes. Theor. Ecol. 7, 101-113 (2014)

28. Pazy, A.: Semigroups of Linear Operators and Applications to Partial Differential Equations, Applied Mathematical Sciences. Springer, New York (1983)

29. Pechersky, E.A., Zhukov, Y.: Uniqueness of Gibbs state for non- ideal gas in $\mathbf{R}^{d}$ : the case of pair potentials. J. Stat. Phys. 97, 145-172 (1999)

30. Riccardi, L.M.: Stochastic population theory: birth and death processes. In: Hallamand, T.G., Levin, S.A. (eds.) Math. Ecol., pp. 155-190. Springer, Berlin (1986)

31. Ruelle, D.: Statistical Mechanics: Rigorous Results. W. A. Benjamin Inc., New York (1969)

32. Thieme, H.R., Voigt, J.: Stochastic semigroups: their construction by perturbation and approximation. In: Weber, M.R., Voigt, J. (eds.) Positivity IV-Theory and Applications, pp. 135-146. Tech. Univ. Dresden, Dresden (2006) 